\title{
1,8-Dipyrenylnaphthalenes: Syntheses, Molecular Structure, and Spectroscopic Properties
}

\author{
Peter Wahl, Claus Krieger, Dieter Schweitzer, and H. A. Staab* \\ Max-Planck-Institut fur medizinische Forschung, Abteilung Organische Chemie, \\ and Abteilung Molekulare Physik, \\ Jahnstr. 29, D-6900 Heidelberg
}

Received March 31, 1983

Syntheses of the 1,8-dipyrenylnaphthalenes $\mathbf{1 - 3}$ are reported. The stereoisomers 1 and 2 were separated; their structural assignment is based on ${ }^{1} \mathrm{H} N \mathrm{NMR}$, on the optical activity of 2 , and on $\mathrm{X}$-ray structure analyses of $\mathbf{1}$ and 2 . Kinetic parameters for the isomerisation $\mathbf{2} \rightleftharpoons \mathbf{1}$ were determined by optical rotation measurements. - Emission spectra of $\mathbf{1 - 3}$ are discussed in comparison to monopyrenyl compounds $\mathbf{4}$ and $\mathbf{8}$. For $\mathbf{1}$ and $\mathbf{3}$ typical 'excimer-like' fluorescence is observed. The difference between 1 and 2 clearly demonstrates the dependence of excimer interactions between the pyrene units on the mutual orientation of the $\pi$-systems involved. - On the basis of $\mathrm{X}$-ray analyses the molecular structures of $\mathbf{1 - 3}$ are discussed with emphasis on $\pi \cdots \pi$-interactions between the pyrene units.

\section{1,8-Dipyrenylnaphthaline: Synthesen, Molekülstruktur und spektroskopische Eigenschaften}

Synthesen der 1,8-Dipyrenylnaphthaline 1-3 werden mitgeteilt. Die Stereoisomeren 1 und 2 wurden getrennt; ihre strukturelle Zuordnung basiert auf ' $\mathrm{H}-\mathrm{NMR}$, auf der optischen Aktivitat von 2 und auf den Rontgenstrukturanalysen von 1 und 2. Kinetische Parameter fur die Isomerisierung $2 \rightleftharpoons 1$ wurden durch Messung der optischen Rotationen bestimmt. - Emissionsspektren von $\mathbf{1 - 3}$ werden im Vergleich zu den Monopyrenyl-Verbindungen $\mathbf{4}$ und $\mathbf{8}$ diskutiert. Fur $\mathbf{1}$ und $\mathbf{3}$ wird eine typische „excimeren-artige“ Fluoreszenz beobachtet. Der Unterschied zwischen 1 und 2 demonstriert deutlich die Abhangigkeit der Excimeren-Wechselwirkung zwischen den Pyren-Einheiten von der gegenseitigen Orientierung der beteiligten $\pi$-Systeme. - Auf der Grundlage von Rontgen-Strukturanalysen werden die Molekülstrukturen von 1-3 im Hinblick auf die $\pi \cdots \pi$ Wechselwirkung zwischen den Pyren-Einheiten diskutiert.

For reasons discussed in the preceding paper on pyrenophanes" excimer models were of interest which contain in an intramolecular system two pyrene units fixed in welldefined sterical arrangements. As a different approach to this problem the syntheses of 1,8-di(1-pyrenyl)naphthalene, of which two stereoisomers 1 and 2 with different overlap of the pyrene units were to be expected, and of 1,8-di(2-pyrenyl)naphthalene 3 were considered.

\section{1,8-Di(1-pyrenyl)naphthalenes $(1,2)$}

Synthesis, Separation, and Structural Assignment of 1 and 2: The synthesis of 1 and 2 was achieved in analogy to the synthesis of 1,8-diphenylnaphthalene ${ }^{2)}$. The Grignard reagent prepared from 1 -bromopyrene ${ }^{3)}$ in tetrahydrofuran was reacted with 1,8-di-

(c) Verlag Chemie GmbH, D-6940 Weinheim, 1984

$0009-2940 / 84 / 0101-0260 \$ 02.50 / 0$ 
iodonaphthalene $\mathrm{e}^{4)}$ in the presence of catalytic amounts of bis(acetylacetonato)nickel(II). In addition to the 1,8(di-1-pyrenyl)naphthalenes $1 / 2$ (26.5\% yield) 1 -(1-pyrenyl)naphthalene (4) and 8,8'-di(1-pyrenyl)-1,1'-binaphthalene (5) were obtained as by-products as well as the known 1,1'-bipyrene ${ }^{5)}$ (for details see Exp. Part).

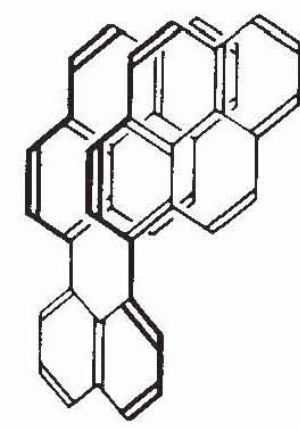

1

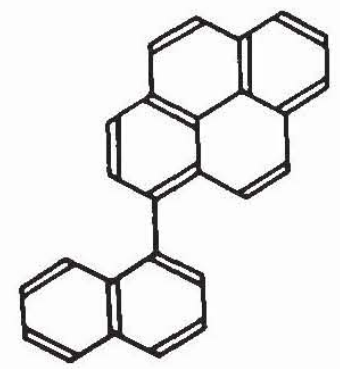

4
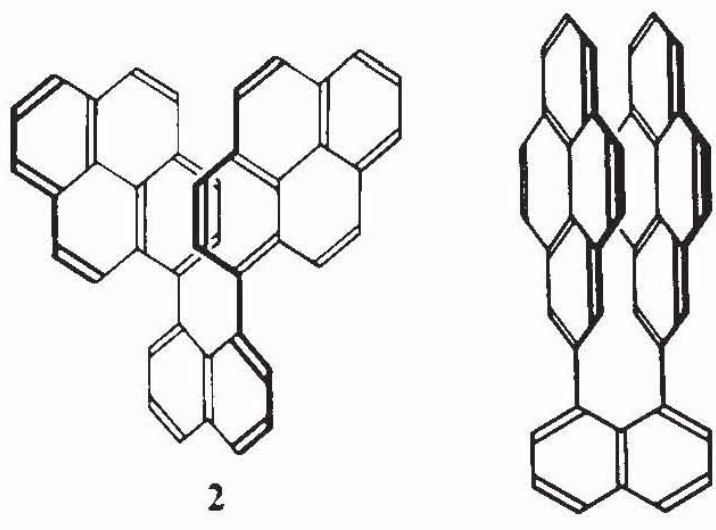

3

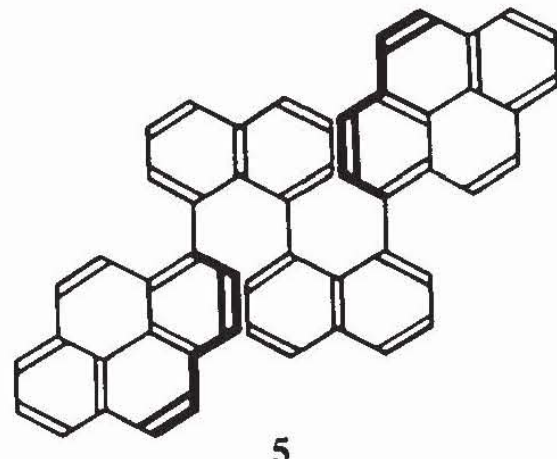

5

The rotamers 1 and 2 were separated by pressure-chromatography on silica from tetrachloromethane and crystallized from dichloromethane/toluene. Analytical and spectroscopie data support the structures suggested [MS: $1: \mathrm{m} / z=528\left(100 \%, \mathrm{M}^{+}\right)$, $326(8), 264\left(10, \mathrm{M}^{2+}\right) ; 2: m / z=528\left(100 \%, \mathrm{M}^{+}\right), 326(9), 264\left(14, \mathrm{M}^{2+}\right) .-{ }^{1} \mathrm{H}$ NMR ( $360 \mathrm{MHz},\left[\mathrm{D}_{2}\right]-1,1,2,2$-tetrachloroethane): $1: \delta=6.947$ and 7.038 (AB, $J=$ $9.1 \mathrm{~Hz}, 4 \mathrm{H}), 7.26-7.40(\mathrm{~m}, 10 \mathrm{H}), 7.450(\mathrm{dd}, J=6.8$ and $1 \mathrm{~Hz}, 2 \mathrm{H}), 7.588(\mathrm{t}, J=$ $7.7 \mathrm{~Hz}, 2 \mathrm{H}), 7.64-7.72(\mathrm{~m}, 4 \mathrm{H}), 8.182(\mathrm{dd}, J=8.4$ and $1 \mathrm{~Hz}, 2 \mathrm{H}) ; 2: \delta=6.201$ and $7.009(\mathrm{AX}, J=7.7 \mathrm{~Hz}, 4 \mathrm{H}), 6.734$ and $7.548(\mathrm{AX}, J=9.6 \mathrm{~Hz}, 4 \mathrm{H}), 7.40-7.43(\mathrm{~m}$, $4 \mathrm{H}), 7.679(\mathrm{t}, 2 \mathrm{H}, J=7.7 \mathrm{~Hz}), 7.841(\mathrm{~d}, J=8.9 \mathrm{~Hz}, 2 \mathrm{H}), 7.98-8.02(\mathrm{~m}, 2 \mathrm{H})$, $8.09-8.15(\mathrm{~m}, 4 \mathrm{H}), 8.184(\mathrm{~d}, J=8.4 \mathrm{~Hz}, 2 \mathrm{H})]$.

The assignment of the structures 1 and 2 to the two separated rotamers was first based on the ${ }^{1} \mathrm{H}$ NMR data: One of the two isomers shows a signal with a striking highfield-shift $(\delta=6.201,2 \mathrm{H})$ which can be explained by assigning this signal to the 3 -protons of the pyrenyl group of the trans-rotamer. These protons are located close to the center of the neighbouring pyrene unit and, thus, are shielded most effectively by the anisotropy of the aromatic system.

This structural assignment has been confirmed by X-ray structure analyses of $\mathbf{1}$ and 2 (see later). It is further established conclusively by the fact that 2 , being a chiral compound unlike $\mathbf{1}$, has been obtained optically active as discussed below. 
cis-trans-Isomerisation $1 \rightleftharpoons 2:{ }^{1} \mathrm{H}$ NMR spectra reveal that in solution an interconversion of 1 and 2 occurs above $60^{\circ} \mathrm{C}$. By integration of specific ${ }^{1} \mathrm{H}$ NMR signals the equilibrium reached both from pure 1 and from pure 2 was determined at 80 and $120^{\circ} \mathrm{C}$ to be approximately $1: 1$.

For establishing kinetic parameters for the interconversion of 1 and 2 use has been made of the crystallographic result that 2 crystallizes in the chiral space group $P 2_{1}$. Thus, a separation of enantiomers was possible by picking out single crystals. For 5 single crystals of $2(0.8$ to $2.1 \mathrm{mg})$ absolute values of specific rotations $[\alpha]_{\mathrm{D}}^{20^{\circ} \mathrm{C}}$ varied between 1930 and $2050^{\circ}$ (in dichloromethane).

By determining the decrease of the optical rotations with time the rate constants for the trans-cis-isomerisation were measured in the temperature range $60-120^{\circ} \mathrm{C}$ (in 1,1,2,2,-tetrachloroethane, Table 1). From these measurements an Arrhenius plot was obtained from which the activation energy $E_{\mathrm{A}}=24.8 \mathrm{kcal} / \mathrm{mol}$ and the preexponential factor $A=4.07 \cdot 10^{12}$ were derived. These data are in rather good correspondence with results obtained for other 1,8-diarylnaphthalenes ${ }^{6}$.

Table 1. Rate Constants $k_{1}$ [min ${ }^{-1}$ ] of Isomerisation $2 \rightleftharpoons 1$ as Determined by Optical Rotation Measurements (in 1,1,2,2-Tetrachloroethane)

\begin{tabular}{c|cc|c}
\hline$T(\mathrm{~K})$ & $10^{4} \cdot k_{1}\left[\mathrm{~min}^{-1}\right]$ & $T(\mathrm{~K})$ & $10^{4} \cdot k_{1}\left[\mathrm{~min}^{-1}\right]$ \\
\hline 333 & 2.182 & 373 & 120.4 \\
343 & 6.355 & 383 & 291.9 \\
353 & 18.04 & 393 & 652.2 \\
363 & 46.72 & & \\
\hline
\end{tabular}

Synthesis of 1-(1-Pyrenyl)naphthalene (4): As reference compound for the spectroscopic comparison with the excimer models 1 and $\mathbf{2}$ compound $\mathbf{4}$ with only one pyrenyl group was prepared from 1-naphthylmagnesium bromide and 1-iodopyrene ${ }^{7)}$ [bis(acetylacetonato)nickel(II), tetrahydrofuran; 62\%]. Analytical and spectroscopic data (see Exp. Part) are in agreement with the structure suggested.

Emission Spectra of 1, 2, and 4: Fluorescence spectra of cis- and trans-1,8-di(1pyrenyl)naphthalenes ( $\mathbf{1}$ and $\mathbf{2}$ ) are shown in Figure 1 together with the fluorescence of the monopyrenylnaphthalene 4 . Whereas for 4 with regard to wavelength and vibrational structure the normal fluorescence of a pyrene chromophore is observed, we see characteristic changes in the fluorescence spectra of the dipyrenyl systems $\mathbf{1}$ and $\mathbf{2}$. As compared to $\mathbf{4}$ the trans-isomer $\mathbf{2}$ shows a fluorescence band which is only moderately red-shifted and still has some vibrational structure. In contrast to 2 , however, the cisisomer 1 has a typical 'excimer' emission with strong red-shift $\left(\Delta v=5800 \mathrm{~cm}^{-1}\right)$ and complete loss of vibrational structure. This remarkable difference between the stereoisomers 1 and 2 must be due to the fact that in 1 we have an optical face-to-face arrangement of the two pyrene units whereas in $\mathbf{2}$ only a rather limited overlap of partial regions of the pyrene $\pi$-systems is possible. Comparison of the absorption spectra of $\mathbf{1}$ and $\mathbf{2}$, on the other hand, shows only minor differences, namely for $\mathbf{1}$ a small red-shift and a slight broadening of the absorption band. The strong Stokes shift, 
which for $\mathbf{1}$ leads to a complete spectral separation of absorption and emission, must therefore be due to an 'excimer'-like interaction between the pyrene units in the excited state. The differences in the fluorescence between $\mathbf{1}$ and $\mathbf{2}$, then, prove conclusively that excimer formation is strongly dependent on the mutual orientation of the $\pi$-systems involved.

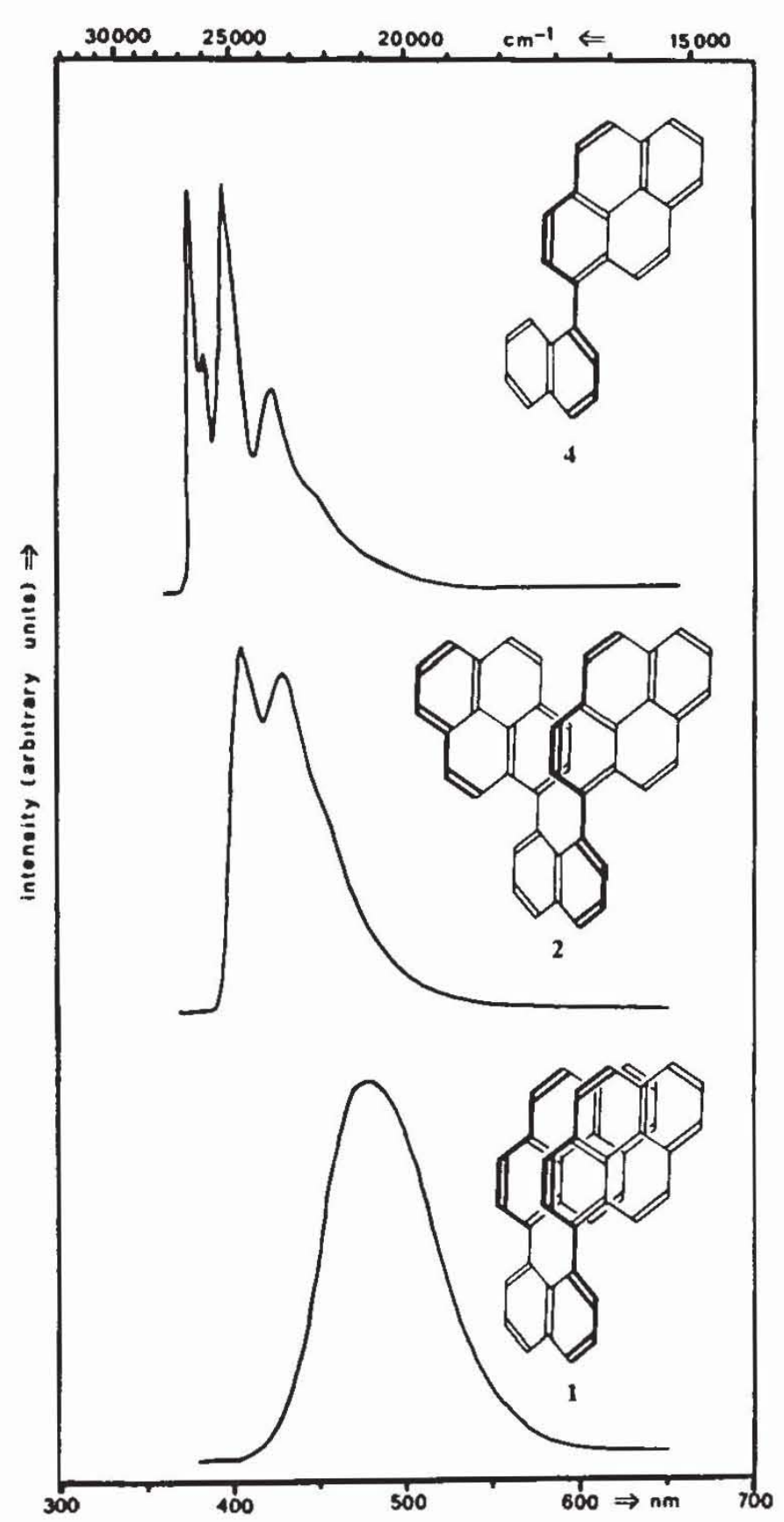

Figure 1. Fluorescence Emission of 1, 2, and 4 (in Methylcyclohexane, 1.3 K)

In contrast to the fluorescence, the phosphorescence emission of both $\mathbf{1}$ and $\mathbf{2}$ is very similar in spectral position and vibrational structure to the phosphorescence of the reference compound $\mathbf{4}$. This means that in the triplet state even of $\mathbf{1}$ there is only very small interaction of the pyrene units as was also found in the series of pyrenophanes ${ }^{1}$. 


\section{Synthesis and Emission Spectra of 1,8-Di(2-pyrenyl)naphthalene (3)}

Synthesis of 3: The synthesis of 3 started from 2-bromo-4,5,9,10-tetrahydropyrene (6), the preparation of which from [2.2]metacyclophane ${ }^{8)}$ was considerably improved. The Grignard reagent from 6 was reacted with 1,8-diiodonaphthalene in the presence of bis(acetylacetonato)nickel(II) to give 1,8-bis(4,5,9,10-tetrahydro-2-pyrenyl)naphthalene (7) in $22 \%$ yield; as by-product of this reaction $4,4^{\prime}, 5,5^{\prime}, 9,9^{\prime}, 10,10^{\prime}$-octahydro- $2,2^{\prime}$ bipyrene was isolated. By dehydrogenation of 7 with 2,3-dichloro-5,6-dicyano-1,4-benzoquinone (DDQ) in toluene 3 was obtained ( $83 \%$ ).<smiles>Brc1cc2c3c(c1)CCc1cccc(c1-3)CC2</smiles>

6

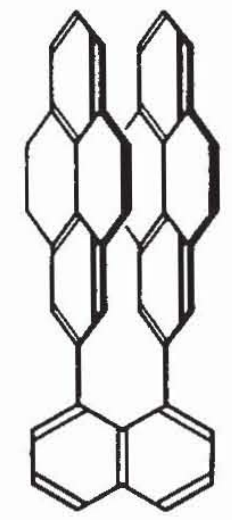

7<smiles>c1ccc2c(-c3cc4ccc5cccc6ccc(c3)c4c56)cccc2c1</smiles>

8

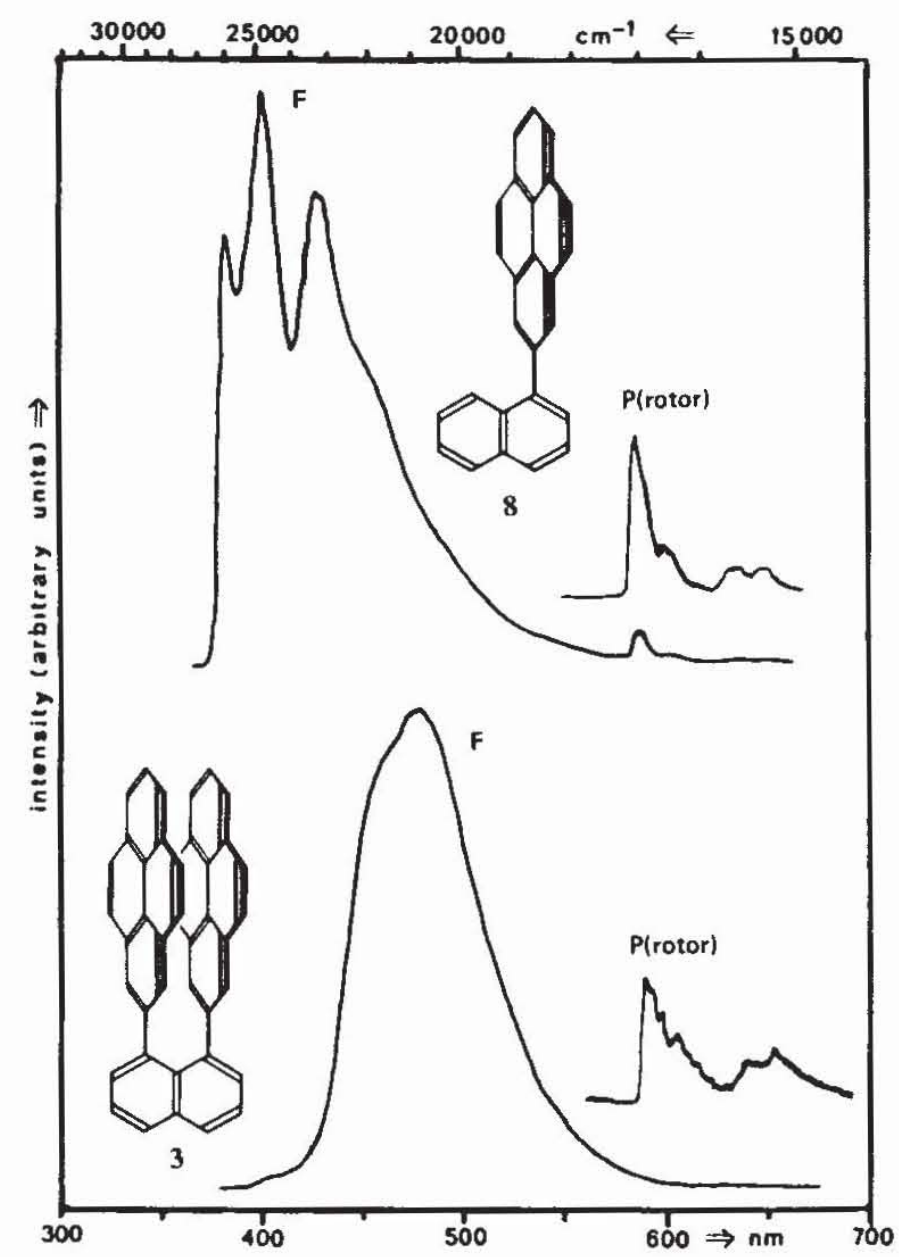

Figure 2. Fluorescence (F) and Phosphorescence (P) of 3 and 8 (in Methylcyclohexane, 1.3 K) 
3 forms pale-yellow crystals (m. p. $331-333^{\circ} \mathrm{C}$ ). Elemental analysis and spectroscopic data are in accordance with the assumed structure [MS: $m / z=528(100 \%$, $\mathrm{M}^{+}$), $326(16), 264\left(9, \mathrm{M}^{2+}\right)$. - ${ }^{1} \mathrm{H}$ NMR (360 MHz, [ $\left.\mathrm{D}_{2}\right]-1,1,2,2$-tetrachloroethane): $\delta=7.261(\mathrm{~s}, 8 \mathrm{H}), 7.51-7.54(\mathrm{~m}, 8 \mathrm{H}), 7.575-7.615(\mathrm{~m}, 2 \mathrm{H}), 7.63-7.66(\mathrm{~m}, 4 \mathrm{H})$, $8.08-8.12(\mathrm{~m}, 2 \mathrm{H})]$. The molecular structure of 3 has been determined by X-ray analysis (see below).

Synthesis of 1-(2-Pyrenyl)naphthalene (8): Derivative $8^{9)}$ which was needed as reference compound for spectroscopic comparison with 3 was synthesized in analogy to 4 by reacting 2-iodopyrene ${ }^{7)}$ with 1-naphthylmagnesium bromide in the presence of bis(acetylacetonato)nickel(II) (tetrahydrofuran, $-10^{\circ} \mathrm{C} ; 54 \%$ ).

Emission Spectra of 3 and 8: Figure 2 shows the emission spectra (in methylcyclohexane, $1.3 \mathrm{~K}$ ) of $\mathbf{3}$ and $\mathbf{8}$. For 8 a fluorescence band is observed which with regard to wavelength and vibrational structure is very similar to the emission of the analogous 1-pyrenylnaphthalene 4 (Figure 1). For both compounds the emission is likely to be essentially due to the pyrene chromophore; although for $\mathbf{8}$ conjugative interaction with the naphthalene $\pi$-system is sterically less hindered a similar torsion around the $\mathrm{Ar}-\mathrm{Ar}$ bond in 4 and 8 has to be assumed which is also indicated by the very similar absorption spectra.

In comparison to $\mathbf{8}$ the dipyrenyl compound $\mathbf{3}$ shows a strongly red-shifted, broad, and structureless emission band. Obviously the similarity of the emission spectra of 3 and 1 must be attributed to the analogous mutual orientations of the pyrene systems in both compounds.

As in the case of compounds 1, 2, and 4, for 3 and 8 almost identical phosphorescence spectra were observed. This means that the triplet state is not significantly affected by an interaction between the two pyrene units in 3 .

\section{Molecular Structures of $1-3^{10)}$}

$X$-Ray Structure Analysis: Crystal and data collection parameters for 1-3 are listed in Table 2 . Intensity data were collected by using a graphite-monochromated Mo- $K_{\alpha}$ radiation and applying $\Theta / 2 \Theta$-scan technique. The structures were solved by conventional direct methods (MULTAN) and were refined by full-matrix least-squares technique using anisotropic temperature factors for non-hydrogen atoms and isotropic temperature factors for hydrogen atoms. Atomic coordinates and thermal parameters for non-hydrogen atoms are given in Tables 3-5 (for the numbering of atoms cf. Figures 3-5).

Molecular Structure of 1: In Figure 3A a view is shown in the direction onto the naphthalene plane which, however, is inclined against the drawing plane by about $20^{\circ}$ by rotation around the $C(4 \mathrm{~A})-\mathrm{C}(8 \mathrm{~A})$ axis (since otherwise only the lateral edges of the pyrenes were to be seen). Figure $3 \mathrm{~B}$ represents a side-view of the molecule in the naphthalene plane perpendicular to $\mathrm{C}(4 \mathrm{~A})-\mathrm{C}(8 \mathrm{~A})$.

In the projection on the naphthalene plane the peri-bonds $\mathrm{C}(1)-\mathrm{C}\left(1^{\prime}\right)$ and $\mathrm{C}(8)-$ $\mathrm{C}\left(1^{\prime \prime}\right)$ are spread for $13^{\circ}$. Furthermore, these bonds deviate from the naphthalene plane for 7 and $9^{\circ}$ in an opposite up-and-down version as, to a minor extent, $C(1)$ and $C(8)$ do (Figure 3B). Due to these deformations the bond angles $C\left(1^{\prime}\right)-C(1)-C(8 A)$, $C(1)-C(8 A)-C(8)$, and $C(8 A)-C(8)-C\left(1^{\prime \prime}\right)$ are increased to $123.2,125.4$, and $124.4^{\circ}$, respectively. The result is a widening of distances from $255 \mathrm{pm}$ for $\mathrm{C}(1) \cdots \mathrm{C}(8)$

Chem. Ber. $117(1984)$ 
Table 2. Crystal and Data Collection Parameters for 1-3

\begin{tabular}{llll}
\hline Compound & 1 & 2 & 3 \\
\hline Formula & $\mathrm{C}_{42} \mathrm{H}_{24}$ & $\mathrm{C}_{42} \mathrm{H}_{24}$ & $\mathrm{C}_{42} \mathrm{H}_{24}$ \\
Molecular mass & 528.6 & 528.6 & 528.6 \\
$a$ [pm] & $2030.1(3)$ & $858.1(1)$ & $1144.8(2)$ \\
$b[\mathrm{pm}]$ & $1141.4(1)$ & $1097.1(1)$ & $1079.0(2)$ \\
$c$ [pm] & $1157.2(1)$ & $1465.5(2)$ & $2154.4(4)$ \\
$\beta\left[^{\circ}\right]$ & $98.89(1)$ & $92.50(2)$ & $93.50(3)$ \\
Space group & $P 2_{1} / c$ & $P 2_{1}$ & $P 2{ }_{1} / n$ \\
$Z$ & 4 & 2 & 4 \\
$D_{\mathrm{x}}$ [gcm $\left.{ }^{-3}\right]$ & 1.32 & 1.27 & 1.32 \\
Max. sin $\Theta / \lambda\left[\mathrm{nm}^{-1}\right]$ & 6.78 & 6.78 & 6.64 \\
No. of reflections & 7009 & 3490 & 6705 \\
$\quad$ measured & 3837 & 1824 & 2309 \\
No. of reflections & & & \\
$\quad$ with $I \geqslant 1.96 \sigma(I)$ & 0.040 & 0.038 & 0.043 \\
$R$ & & & \\
\hline
\end{tabular}

A
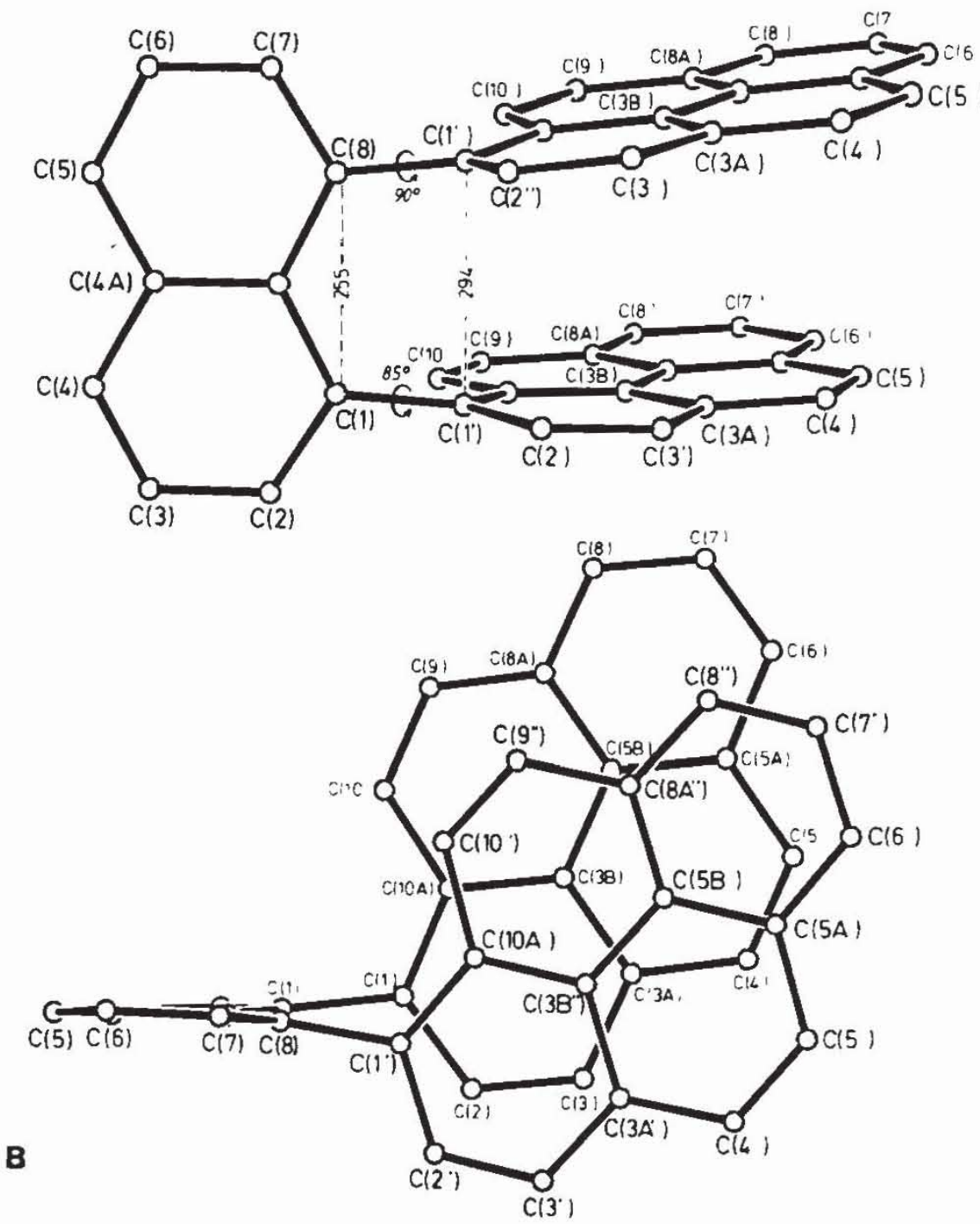

Figure 3. Molecular Structure of $\mathbf{1}$ in a View onto the Naphthalene Plane (A) and in a View Parallel to the Naphthalene Plane (B) (for Details see Text) 


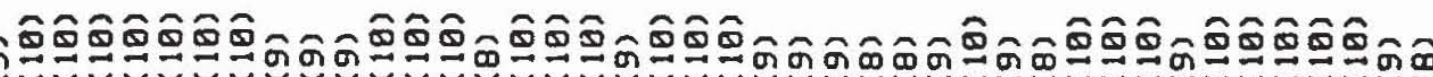

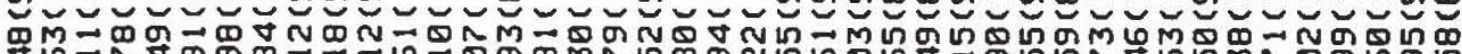

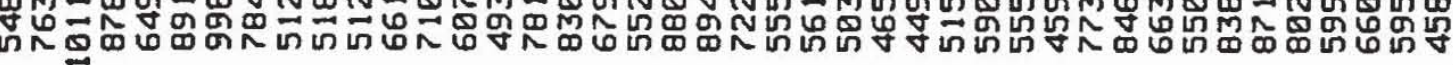

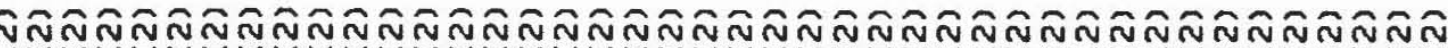

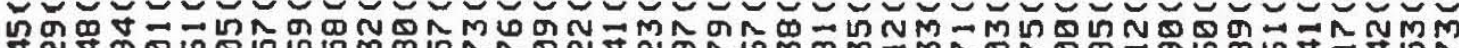
寸ํ. m MV N

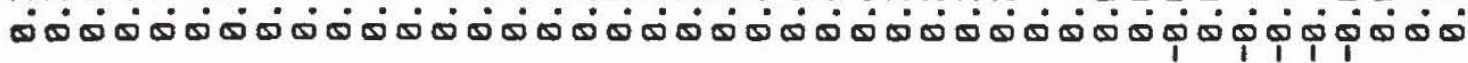

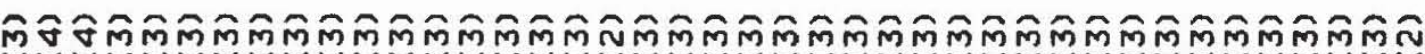
N -

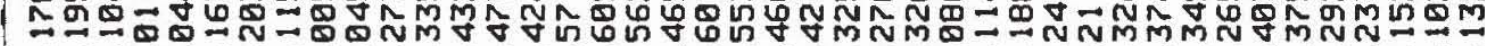

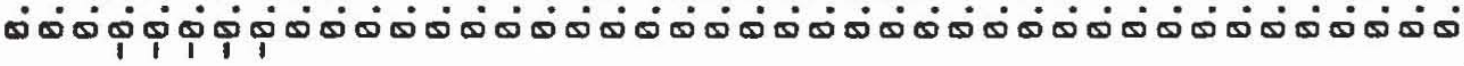

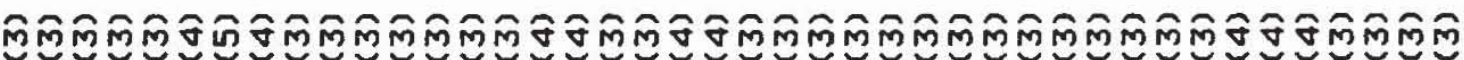
\%N.

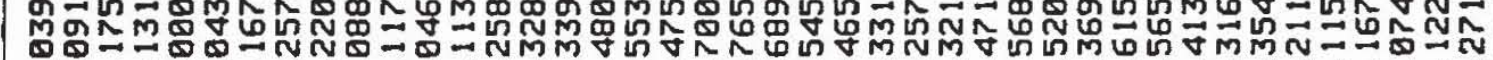
बiि

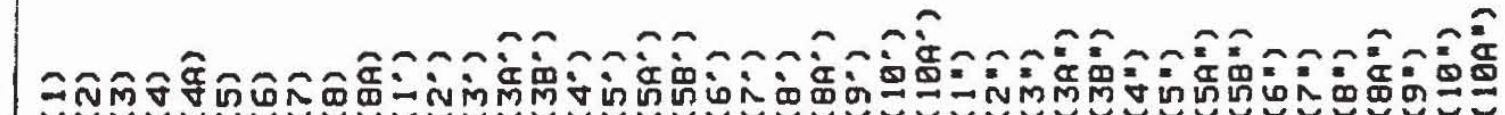

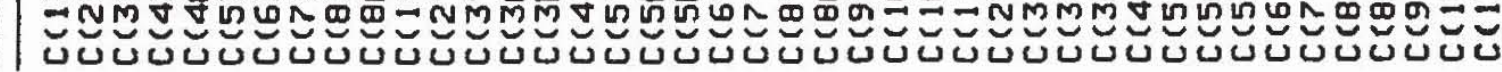

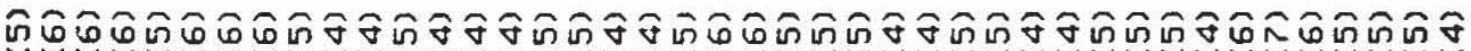
onNư

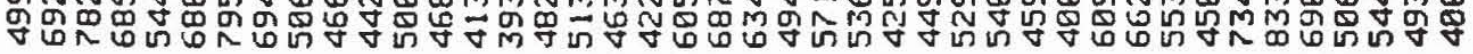

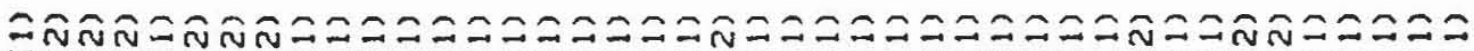

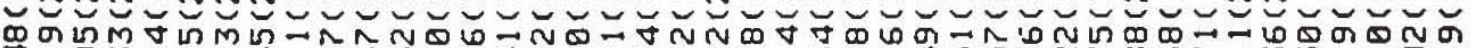

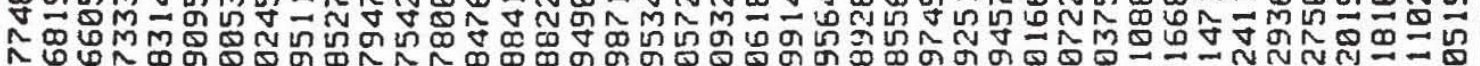

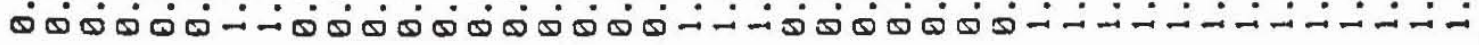

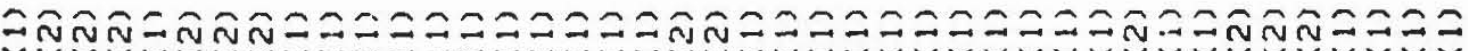

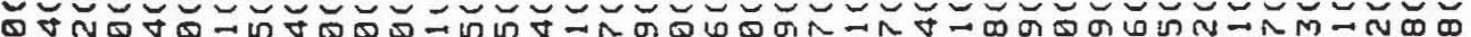

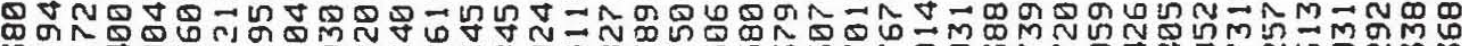
Mี Q Q

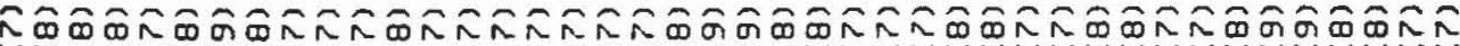

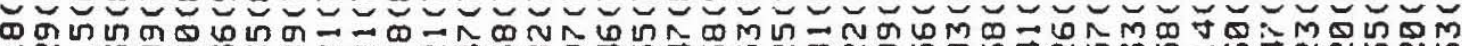

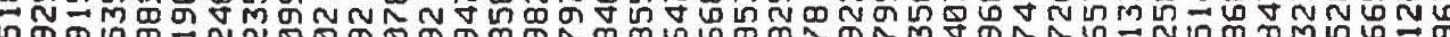

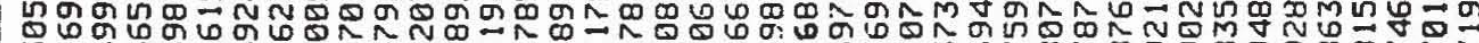

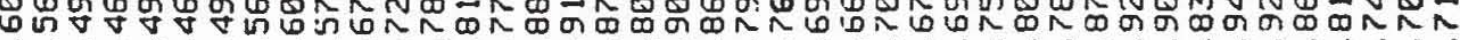

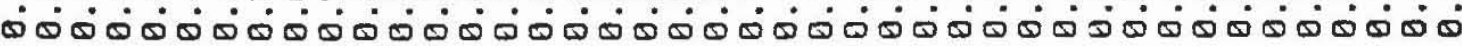

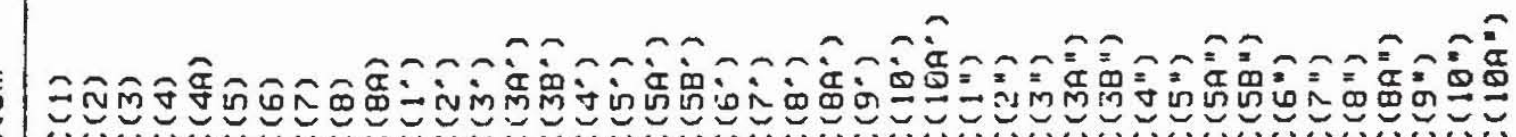
| 
Table 5. Atomic Coordinates and Thermal Parameters $U_{\text {equ. }}$ (in $\mathrm{pm}^{2}$ ) for 3 (Numbers in Parentheses are Estimated Standard Deviations in Units of the Last Quoted Digit)

\begin{tabular}{|c|c|c|c|c|}
\hline Atom & $x$ & 4 & $z$ & Uoau. \\
\hline$c(1)$ & $-0.1572(3)$ & $0.4964(4)$ & $0.0698(2)$ & $523(18)$ \\
\hline$C(2$ & $-0.2125(4)$ & $0.5501(4)$ & $0.0189(2)$ & $689(20)$ \\
\hline$c(3)$ & $-0.2376(4)$ & $0.4886(5)$ & $-0.0379(2)$ & $793(28)$ \\
\hline$c(4)$ & $-0.2068(4)$ & $0.3655(4)$ & $-0.0410(2)$ & $760(20)$ \\
\hline$C(4 A)$ & $-0.1473(3)$ & 0.3047 (4) & $0.0090(2)$ & $607(10)$ \\
\hline$c(5)$ & $-0.1183(4)$ & $0.1762(4)$ & 0.6030 (2) & $706(20)$ \\
\hline$[(6)$ & $-0.0648(4)$ & $0.1155(4)$ & $8.0504(2)$ & $754(20)$ \\
\hline$c(7)$ & $-0.0311(3)$ & 0.1766 (4) & $0.1961(2)$ & $647(10)$ \\
\hline$C(8)$ & $-0.0532(3)$ & $0.3020(3)$ & $0.1146(2)$ & $486(9)$ \\
\hline$C(B A)$ & $-0.1170(3)$ & $0.3680(4)$ & $0.0666(2)$ & $522(18)$ \\
\hline$C\left(1^{\circ}\right)$ & $-0.1927(3)$ & $0.5348(4)$ & $0.1831(2)$ & $496(9)$ \\
\hline$c\left(2^{\circ}\right)$ & $-0.1458(3)$ & $0.5710(4)$ & $0.1275(2)$ & $504(9)$ \\
\hline$c\left(3^{\circ}\right)$ & $-0.0836(3)$ & $0.6837(4)$ & $0.1276(2)$ & $54 i(10)$ \\
\hline$C\left(3 A^{\circ}\right)$ & $-0.0640(3)$ & $0.7535(4)$ & $0.1807(2)$ & $501(10)$ \\
\hline$C\left(38^{\circ}\right)$ & $-0.1062(3)$ & $0.7106(3)$ & $0.2372(2)$ & $444(8)$ \\
\hline$C\left(4^{\circ}\right)$ & $0.0051(3)$ & $0.8651(4)$ & $0.1814(2)$ & $639(18)$ \\
\hline$\left[\left(5^{\circ}\right)\right.$ & $0.0293(3)$ & $0.9266(4)$ & $0.2346(2)$ & $683(20)$ \\
\hline$C\left(5 A^{\circ}\right)$ & $-0.0107(3)$ & 0.8855 (4) & $0.2929(2)$ & $576(10)$ \\
\hline$c\left(5 B^{\circ}\right)$ & $-0.0799(3)$ & $0.7756(3)$ & $0.2925(2)$ & $478(9)$ \\
\hline$c\left(6^{\circ}\right)$ & $0.0137(4)$ & $0.9467(4)$ & $0.3484(2)$ & $760(2 \theta)$ \\
\hline$C\left(7^{\circ}\right)$ & $-0.0245(4)$ & $0.9045(4)$ & $0.4050(2)$ & $796(28)$ \\
\hline$c\left(\theta^{\circ}\right)$ & $-0.0920(3)$ & 0.7965 (4) & $0.4045(2)$ & $703(20)$ \\
\hline$C\left(B A^{\circ}\right)$ & $-0.1195(3)$ & $0.7316(4)$ & 0.3499 (2) & $571(9)$ \\
\hline$C\left(9^{\circ}\right)$ & $-0.1868(3)$ & $0.6171(4)$ & $0.3481(2)$ & $639(10)$ \\
\hline$c\left(10^{\circ}\right)$ & $-0.2153(3)$ & $0.5579(4)$ & $0.2952(2)$ & $548(9)$ \\
\hline$C\left(10 A^{\circ}\right)$ & $-0.1740(3)$ & 0.6006 & 0.237 & $436(8)$ \\
\hline$C(1 ")$ & $-0.0347(3)$ & $0.2984(4)$ & $0.2307(2)$ & $532(10)$ \\
\hline$c\left(2^{\prime \prime}\right)$ & $-0.0057(3)$ & 0.3523 (4) & $0.1748(2)$ & $479(9)$ \\
\hline$C\left(3^{\prime \prime}\right)$ & $0.0730(3)$ & $0.4518(4)$ & $0.1782(2)$ & $503(10)$ \\
\hline$C\left(3 A^{\circ}\right)$ & $0.1159(3)$ & $0.4994(4)$ & $0.2340(2)$ & $488(9)$ \\
\hline$C\left(3 B^{n}\right)$ & $0.0831(3)$ & $0.4452(3)$ & 0.296 & $480(9)$ \\
\hline$C\left(4^{\prime \prime}\right)$ & $0.1905(3)$ & $0.6003(4)$ & $0.2385(2)$ & $589(9)$ \\
\hline$c\left(5^{\circ}\right)$ & $0.2244(3)$ & 0.6568 (4) & 0.2937 (2) & $683(20)$ \\
\hline$C(5 A *)$ & $0.1938(3)$ & $0.6038(4)$ & $0.3511(2)$ & $605(10)$ \\
\hline$C\left(5 B^{\circ}\right)$ & $0.1218(3)$ & $0.4964(4)$ & $0.3483(2)$ & $549(9)$ \\
\hline$C\left(6^{n}\right)$ & $0.2295(4)$ & $0.6532(4)$ & $0.4981(2)$ & $763(28)$ \\
\hline$C\left(7^{\circ}\right)$ & $.1985(4)$ & $0.5989(4)$ & $0.4621(2)$ & $891(2 y)$ \\
\hline$C\left(8^{\circ}\right)$ & $.1284(4)$ & $0.4927(5)$ & $0.4611(2)$ & $822(20)$ \\
\hline$C\left(B A^{\prime \prime}\right)$ & $0895(3)$ & $0.4415(4)$ & $0.4046(2)$ & $647(10)$ \\
\hline$C\left(9^{\circ}\right)$ & $0.0142(4)$ & $0.3334(4)$ & $0.400 E(2)$ & $744(28)$ \\
\hline$c\left(10^{\circ}\right)$ & $-0.0263(4)$ & $0.2900(4)$ & $0.3455(2)$ & $680(10)$ \\
\hline$c(10)$ & $0.0866(3)$ & $0.3416(4)$ & $0.2876(2)$ & $509(9)$ \\
\hline
\end{tabular}

to $294 \mathrm{pm}$ for $\mathrm{C}\left(1^{\prime}\right) \cdots \mathrm{C}\left(1^{\prime \prime}\right)$. The pyrene systems show a deviation from planarity for $\mathrm{C}\left(1^{\prime}\right)$ and $\mathrm{C}\left(1^{\prime \prime}\right)$ [angles of planes $\mathrm{C}\left(10 \mathrm{~A}^{\prime}\right) / \mathrm{C}\left(1^{\prime}\right) / \mathrm{C}\left(2^{\prime}\right)$ and $\mathrm{C}\left(10 \mathrm{~A}^{\prime \prime}\right) / \mathrm{C}\left(1^{\prime \prime}\right) / \mathrm{C}\left(2^{\prime \prime}\right)$ with the mean planes through all other carbon atoms of the pyrene units: 7.5 and $3.9^{\circ}$, respectively]. The remaining carbon atoms of both pyrene systems are nearly coplanar (maximal deviation from best plane 6 and $2 \mathrm{pm}$ ). The pyrenyl groups are rotated against the naphthalene plane for 85 and $90^{\circ}$. This different torsion together with the spreading of $\mathrm{C}(1)-\mathrm{C}\left(1^{\prime}\right)$ and $\mathrm{C}(8)-\mathrm{C}\left(1^{\prime \prime}\right)$ bonds leads to a relatively strong lateral displacement from an ecliptic arrangement of the two pyrene units the overlap of which nevertheless is considerable. With regard to the 'excimer-like' state of 1 it should be mentioned that the overlap between the two pyrene systems may easily be increased by mere changes of torsional angles. The interplanar distance for the central parts of the pyrenyl groups is about $340 \mathrm{pm}$. Some short distances between carbon atoms of the two pyrene units are listed in Table 6. 
Table 6. Some Short Distances between Carbon Atoms of Different Pyrene Units in 1 (in pm)

\begin{tabular}{llllll}
\hline $\mathrm{C}(1)$ & $\cdots \mathrm{C}\left(1^{\prime \prime}\right)$ & 294 & $\mathrm{C}\left(3 \mathrm{~A}^{\prime}\right) \cdots \mathrm{C}\left(3 \mathrm{~B}^{\prime \prime}\right)$ & 337 \\
$\mathrm{C}(2)$ & $\cdots \mathrm{C}\left(1^{\prime \prime}\right)$ & 307 & $\mathrm{C}\left(3^{\prime}\right) \cdots \mathrm{C}\left(3 \mathrm{~A}^{\prime \prime}\right)$ & 338 \\
$\mathrm{C}\left(2^{\prime}\right)$ & $\cdots \mathrm{C}\left(2^{\prime \prime}\right)$ & 311 & $\mathrm{C}\left(10^{\prime}\right) \cdots \mathrm{C}\left(10^{\prime}\right)$ & 341 \\
$\mathrm{C}\left(10 \mathrm{~A}^{\prime}\right) \cdots \mathrm{C}\left(10^{\prime \prime}\right)$ & 323 & $\mathrm{C}\left(3^{\prime}\right) \cdots \mathrm{C}\left(3^{\prime \prime}\right)$ & 347 \\
$\mathrm{C}(1)$ & $\cdots \mathrm{C}\left(10 \mathrm{~A}^{\prime \prime}\right)$ & 324 & $\mathrm{C}\left(2^{\prime}\right) \cdots \mathrm{C}\left(10 \mathrm{~A}^{\prime \prime}\right)$ & 347 \\
$\mathrm{C}\left(10 \mathrm{~A}^{\prime}\right) \cdots \mathrm{C}\left(10 \mathrm{~A}^{\prime}\right)$ & 331 & $\mathrm{C}\left(2^{\prime}\right) \cdots \mathrm{C}\left(3^{\prime \prime}\right)$ & 349 \\
\hline
\end{tabular}

Table 7. C ... C Bond Lengths of 1 and 2 (in pm; Numbers in Parentheses are Estimated Standard Deviations in Units of the Last Quoted Digit)

\begin{tabular}{|c|c|c|c|c|c|c|}
\hline & & $!$ & 2 & & 1 & 2 \\
\hline$c(1)$ & $-c(2)$ & $137.0(1)$ & $138.4(4)$ & $c\left(6^{\circ}\right)-c\left(7^{\circ}\right)$ & $137.3(1)$ & $135.8(5)$ \\
\hline$C(1)$ & $-C(B A)$ & $143.7(1)$ & $143.1(4)$ & $C\left(7^{\circ}\right)-C\left(8^{\circ}\right)$ & $137.5(1)$ & $137.9(4)$ \\
\hline$c(1)$ & $-c\left(1^{\circ}\right)$ & $149.6(1)$ & $149.7(4)$ & $C\left(B^{\circ}\right)-C\left(B A^{\circ}\right)$ & $139.4(1)$ & $139.6(4)$ \\
\hline$c(2)$ & $-c(3)$ & $140.7(1)$ & $138.8(6)$ & $C\left(B A^{\circ}\right)-C\left(9^{\circ}\right)$ & $142.7(1)$ & $142.4(4)$ \\
\hline$c(3)$ & $-c(4)$ & $133.6(1)$ & $133.3(6)$ & $c\left(9^{\circ}\right)-c\left(10^{\circ}\right)$ & $134.8(1)$ & $134.2(4)$ \\
\hline$C(4)$. & $-C(4 A)$ & $141.1(1)$ & $141.7(5)$ & $C\left(10^{\circ}\right)-C\left(100^{\circ}\right)$ & $143.1(1)$ & $143.7(3)$ \\
\hline$C(49)$ & $-c(5)$ & $141.2(1)$ & $140.5(4)$ & $c\left(1^{\circ}\right)-c\left(2^{*}\right)$ & $138.8(1)$ & $139.5(3)$ \\
\hline$C(4 A)$ & $-C(B A)$ & $143.1(1)$ & $142.4(3)$ & $C\left(1^{\circ}\right)-C\left(10 A^{\circ}\right)$ & $139.8(1)$ & $140.5(3)$ \\
\hline$c(5)$ & $-c(6)$ & $134.5(1)$ & $133.1(5)$ & $c\left(2^{\prime \prime}\right)-c\left(3^{\circ}\right)$ & $137.9(1)$ & $136.9(4)$ \\
\hline$c(6)$ & $-c(7)$ & $140.4(1)$ & $141.3(5)$ & $C\left(3^{\circ}\right)-C\left(3 A^{\circ}\right)$ & $138.4(1)$ & $138.7(4)$ \\
\hline$c(7)$ & $-C(B)$ & $136.3(1)$ & $136.3(4)$ & $C\left(3 A^{\circ}\right)-C\left(3 B^{\circ}\right)$ & $142 . \theta(1)$ & $142.1(3)$ \\
\hline$C(8)$ & $-C(B A)$ & $143.4(1)$ & $143.7(3)$ & $C\left(3 A^{\circ}\right)-C\left(4^{\circ}\right)$ & $143.5(1)$ & $144.8(4)$ \\
\hline$C(\theta)$ & $-\left[\left(1^{\circ}\right)\right.$ & $149.9(1)$ & $149.8(3)$ & $c\left(38^{\circ}\right)-c\left(5 B^{\prime \prime}\right)$ & $142.8(1)$ & $142 . \theta(3)$ \\
\hline$c\left(1^{\circ}\right)$ & $-C\left(2^{\circ}\right)$ & $138.9(1)$ & $139.1(4)$ & $C\left(3 B^{\circ}\right)-C\left(18 A^{\circ}\right)$ & $141.5(1)$ & $141.5(3)$ \\
\hline$C\left(1^{\circ}\right)$ & $-C\left(1 \theta A^{\circ}\right)$ & $140.8(1)$ & $140.7(3)$ & $C\left(4^{\circ}\right)-c\left(5^{\circ}\right)$ & $134.1(1)$ & $133.6(5)$ \\
\hline$\left[\left(2^{\circ}\right)\right.$ & $-c\left(3^{\circ}\right)$ & $137.8(1)$ & $137.1(4)$ & $C\left(5^{\prime}\right)-C\left(5 A^{\circ}\right)$ & $142.6(1)$ & $142.9(4)$ \\
\hline$C\left(3^{\circ}\right)$ & $-C\left(3 A^{\circ}\right)$ & $139.1(1)$ & $139.8(4)$ & $C\left(5 A^{\circ}\right)-C\left(5 B^{\circ}\right)$ & $141.2(1)$ & $142.7(4)$ \\
\hline$C\left(3 A^{\circ}\right)$ & )$-c\left(38^{\circ}\right)$ & $141.6(1)$ & $142.2(4)$ & $C\left(5 A^{\circ}\right)-C\left(6^{\circ}\right)$ & $139.9(1)$ & $138.9(4)$ \\
\hline$C\left(3 A^{\circ}\right)$ & )$-C\left(4^{\circ}\right)$ & $142.4(1)$ & $142.9(4)$ & $C\left(5 D^{\circ}\right)-C\left(B A^{\circ}\right)$ & $141.5(1)$ & $140.2(3)$ \\
\hline$C\left(38^{\circ}\right)$ & )$-C\left(58^{\circ}\right)$ & $142.3(1)$ & $142.7(3)$ & $C\left(6^{\prime \prime}\right)-C\left(7^{\circ}\right)$ & $137.2(2)$ & $135.4(5)$ \\
\hline$C\left(3 B^{\circ}\right)$ & )$-C\left(1 \theta A^{\circ}\right)$ & $142.3(1)$ & $140.5(3)$ & $c\left(7^{\circ}\right)-c\left(\theta^{*}\right)$ & $137.8(2)$ & $139.2(5)$ \\
\hline$C\left(4^{\circ}\right)$ & $-c\left(5^{\circ}\right)$ & $134.7(1)$ & $134.3(4)$ & $C\left(\theta^{*}\right)-C\left(B A^{*}\right)$ & $139.7(1)$ & $140.7(4)$ \\
\hline$\left[\left(5^{\circ}\right)\right.$ & $-C\left(5 A^{\circ}\right)$ & $142.9(1)$ & $142.8(4)$ & $C\left(B A^{\circ}\right)-C\left(9^{*}\right)$ & $142.4(1)$ & $141.6(4)$ \\
\hline$C\left(5 A^{\circ}\right)$ & )$-c\left(58^{\circ}\right)$ & $141.3(1)$ & $142.1(4)$ & $c\left(9^{\circ}\right)-c\left(10^{\circ}\right)$ & $134.4(1)$ & $135.7(4)$ \\
\hline$C\left(5 A^{\circ}\right)$ & )$-C\left(6^{\circ}\right)$ & $139.6(1)$ & $140.7(4)$ & $C\left(10^{\circ}\right)-C\left(10 A^{\circ}\right)$ & $143.4(1)$ & $143.7(3)$ \\
\hline$C\left(5 B^{\circ}\right)$ & )$-C\left(8 A^{\circ}\right)$ & $141.6(1)$ & $141.4(4)$ & & & \\
\hline
\end{tabular}

Chem. Ber. $117(1984)$ 
Bond lengths of 1 are listed in Table 7 together with those of the isomer 2. Bond angles of $\mathbf{1}$ and $\mathbf{2}$ are given in Table 8. With the exception of the deformations mentioned the bond lengths and angles are in the normal range.

Table 8. Bond Angles of 1 and 2 (in ${ }^{\circ}$; Numbers in Parentheses are Estimated Standard Deviations in Units of the Last Quoted Digit)

\begin{tabular}{|c|c|c|c|c|c|c|c|c|c|c|}
\hline & & & $!$ & 2 & & $!$ & 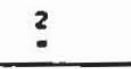 & & $!$ & ? \\
\hline 2) & $-c(1)$ & $C(B A)$ & $119.0(1)$ & $118.8(3)$ & $\left(3 A^{\circ}\right)-C\left(3 B^{\circ}\right)-C\left(5 B^{\circ}\right)$ & $119.4(1)$ & $119.3(3)$ & $\left.2^{\circ}\right)=c\left(3^{\circ}\right)-c$ & $120.9(1)$ & ? \\
\hline (2) & $-c(1)$ & $-\left[\left(1^{\circ}\right)\right.$ & $117.5(1)$ & $5.8(3)$ & $C\left(3 A^{\circ}\right)-c\left(38^{\circ}\right)-C\left(1 B A^{\circ}\right)$ & $128.1(1)$ & $28.8(3)$ & $C\left(3^{\circ}\right)-c\left(3 A^{\circ}\right)-C\left(38^{\circ}\right)$ & $118.9(1)$ & $118.3(3)$ \\
\hline (BA) & $-c(1)$ & $-\left[\left(1^{\circ}\right)\right.$ & $123.3(1)$ & $125.3(2)$ & $C\left(58^{\circ}\right)-c\left(38^{\circ}\right)-C\left(19 A^{\circ}\right)$ & $128.4(t)$ & $119.9(3)$ & $C\left(3^{\circ}\right)-C\left(3 A^{\circ}\right)-C\left(4^{\circ}\right)$ & 122. & 3) \\
\hline (1) & $-c(2)$ & $-c(3)$ & $122.8(1)$ & $122.4(4)$ & $C\left(3 A^{\circ}\right)-C\left(4^{\circ}\right)-C\left(5^{\circ}\right)$ & $121.4(1)$ & $128.9(4)$ & $C\left(38^{\circ}\right)-C\left(3 A^{\circ}\right)-\left[\left(4^{\circ}\right)\right.$ & 118 & $118.6(3)$ \\
\hline (2) & $-c(3)$ & $-c(4)$ & $129.2(1)$ & $128.3(4)$ & $C\left(4^{\circ}\right)-C\left(5^{\circ}\right)-c\left(5 A^{\circ}\right)$ & $121.3(1)$ & $122.8(4)$ & $c\left(3 A^{\circ}\right)-c\left(38^{\circ}\right)-c\left(5 B^{\circ}\right)$ & 119. & $119.7(3)$ \\
\hline (3) & $-C(4)$ & $-c(4 A)$ & 121.8 & $120.6(4)$ & $C\left(5^{\circ}\right)-C\left(5 A^{\circ}\right)-C\left(5 \theta^{\circ}\right)$ & 118.7 & $118.4(3)$ & $c\left(3 A^{\circ}\right)-c\left(3 B^{\circ}\right)-c$ & 119 & $128.8(3)$ \\
\hline$c(4)$ & $-C(4 A)$ & $-c(s)$ & $119.8(1)$ & $119.3(4)$ & $C\left(5^{\circ}\right)-C\left(5 A^{\circ}\right)-C\left(6^{\circ}\right)$ & $122.3(1)$ & $123.8(4)$ & $C\left(58^{\circ}\right)-C\left(38^{\circ}\right)-C\left(10 A^{\circ}\right)$ & 12 & $128.2(3)$ \\
\hline$C(4)$ & $-C(4 A)$ & $-C(8 \beta)$ & 1190 & $128.5(4)$ & $C\left(58^{\circ}\right)-C\left(5 A^{\circ}\right)-C\left(6^{\circ}\right)$ & 119.111 & $17.8(4)$ & 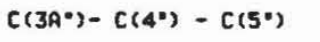 & ) & (4) \\
\hline (5) & $-c(4 A)$ & $-C(B A)$ & 128 & ) & $C\left(3 B^{\circ}\right)-C\left(5 B^{\circ}\right)-C\left(5 A^{\circ}\right)$ & 120 & 3) & $C\left(4^{\circ}\right)-C\left(5^{\circ}\right)-C\left(5 A^{\circ}\right)$ & 12 & (4) \\
\hline$C(4 A)$ & $-c(5)$ & $-c(6)$ & $121.2(1)$ & $121.3(4)$ & $C\left(38^{\circ}\right)-C\left(58^{\circ}\right)-C\left(88^{\circ}\right)$ & $128.1(1)$ & (3) & $C\left(5^{\circ}\right)-C\left(5 A^{\circ}\right)-C\left(5 B^{\circ}\right)$ & 118 & $118.4(3)$ \\
\hline$c(5)$ & $-c(6)$ & $-c(7)$ & $119.1(1)$ & $120.8(4)$ & $C\left(5 A^{\circ}\right)-C\left(5 B^{\circ}\right)-C\left(B A^{\circ}\right)$ & $119.7(1)$ & 3) & $C\left(5^{\circ}\right)-c\left(5 A^{\circ}\right)-C\left(6^{\circ}\right)$ & 122 & $124.8(4)$ \\
\hline$c(6)$ & $-c(7)$ & $-c(\theta)$ & $122.7(1)$ & $21.4(4)$ & $C\left(5 A^{\circ}\right)-C\left(6^{\circ}\right)-C\left(7^{\circ}\right)$ & $128.5(1)$ & $121.7(4)$ & $C\left(58^{\circ}\right)-C\left(5 A^{\circ}\right)-C\left(6^{\circ}\right)$ & 118 & $117.7(4)$ \\
\hline$c(7)$ & $-c(\theta)$ & $-c(B A)$ & $119.5(1)$ & $119.8(3)$ & $C\left(6^{\circ}\right)-C\left(7^{\circ}\right)-C\left(\theta^{\circ}\right)$ & $121.3(1)$ & 121 & $C\left(3 B^{\circ}\right)-C\left(5 B^{\circ}\right)-C\left(5 A^{\circ}\right)$ & $128.8(1)$ & $119.6(3)$ \\
\hline$c(7)$ & $-c(8)$ & $c\left(11^{\circ}\right)$ & $115.6(1)$ & $115.7(3)$ & $C\left(7^{\circ}\right)-C\left(8^{\circ}\right)-C\left(8 A^{\circ}\right)$ & $128.3(1)$ & $128.8(4)$ & $C\left(3 B^{\circ}\right)-c\left(5 B^{\circ}\right)-c\left(B A^{\circ}\right)$ & 119 & $128.3(3)$ \\
\hline$C(B A)$ & $-[(\theta)$ & $-\left(11^{\circ}\right)$ & $124.6(1)$ & $124.5(2)$ & $C\left(5 B^{\circ}\right)-C\left(B A^{\circ}\right)-C\left(B^{\circ}\right)$ & 119 & $119.8(3)$ & $C\left(5 A^{\circ}\right)-C\left(5 \theta^{\circ}\right)-$ & 12 & $120.1(3)$ \\
\hline$c(1)$ & $-C(8 A)$ & $-c(4 a)$ & $117.8(1)$ & $117.4(3)$ & $c\left(5 \theta^{\circ}\right)-c($ & & O(3) & $C\left(5 A^{\circ}\right)$ & & $122.5(4)$ \\
\hline$c(1)$ & $-C(8 A)$ & $-C(B)$ & $125.3(1)$ & $125.3(2)$ & $C\left(\theta^{\circ}\right)-c(B A$ & & 3) & c & & $128.7(4)$ \\
\hline (4A) & $-c(\theta A)$ & $-c(\theta)$ & $116.9(1$ & $117.3(3)$ & C $18 A^{\circ}$ & $121.8(1)$ & ) & ) & & $119.4(4)$ \\
\hline$c(1)$ & $-c\left(1^{\circ}\right)$ & $-c\left(2^{\circ}\right)$ & $121.8(1)$ & $119.3(3)$ & $c\left(9^{\circ}\right)-c\left(18^{\circ}\right)-$ & 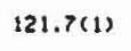 & ) & $C\left(5 B^{\circ}\right)-C\left(B A^{\circ}\right)-$ & & $119.5(4)$ \\
\hline (1) & $-c\left(1^{\prime}\right)$ & $-c\left(18 A^{\circ}\right)$ & $120.3(1)$ & $122.8(3)$ & $C\left(1^{\circ}\right)-C\left(18 A^{\circ}\right)-C\left(38^{\circ}\right)$ & $119.4(1)$ & ) & $\cdot)-c\left(B A^{\circ}\right)-c\left(g^{\circ}\right)$ & $118.5(1)$ & $118.8(3)$ \\
\hline$(2)$ & $-c\left(1^{\prime}\right)$ & $-C\left(18 A^{\circ}\right)$ & $118.8(1)$ & $=0$ & $C\left(1^{\circ}\right)-C\left(18 A^{\circ}\right)-C\left(18^{\circ}\right)$ & $122.8(1)$ & ) & $C\left(8^{\circ}\right)-C\left(\theta^{\circ}\right)-C\left(9^{\circ}\right)$ & 1) & $121.7(3)$ \\
\hline e & בירו) & $-c\left(3^{\circ}\right)$ & $121.8(1)$ & $22.8(3)$ & $C\left(38^{\circ}\right)-C\left(180^{\circ}\right)-C\left(10^{\circ}\right)$ & $117.7(1)$ & 3) & $C\left(B A^{\circ}\right)-C\left(9^{\circ}\right)-C\left(1 \theta^{\circ}\right)$ & 121. & 1.6 \\
\hline (2*) & $-c\left(3^{\circ}\right)$ & $-c\left(3 A^{\circ}\right)$ & $128.9(1)$ & $128.8(3)$ & $c(8)-c\left(1^{\circ}\right)-c\left(2^{\circ}\right)$ & $118.8(1)$ & $119.6(3)$ & $c\left(9^{\circ}\right)-c\left(10^{\circ}\right)-c\left(18 \theta^{\circ}\right)$ & $121.4(1)$ & 1.8 \\
\hline$\left(3^{\circ}\right)$ & $-c\left(3 A^{\circ}\right)$ & )$-c\left(38^{\circ}\right)$ & $118.5(1)$ & $117.9(3)$ & $C(B)-C\left(1^{\circ}\right)-C\left(18 A^{\circ}\right)$ & $122.3(1)$ & $121.7(2)$ & $C\left(1^{\circ}\right)-C\left(18 A^{\circ}\right)-C\left(3 \theta^{\circ}\right)$ & $119.5(1)$ & 19.8 \\
\hline$\left(3^{\circ}\right)$ & $-\left[\left(3 A^{\circ}\right)\right.$ & )$-c\left(4^{\circ}\right)$ & $122.4(1)$ & $122.8(3)$ & $C\left(2^{\circ}\right)-\left[\left(1^{\circ}\right)-C\left(10 A^{\circ}\right)\right.$ & ) & $118.7(3)$ & $C\left(1^{\circ}\right)-C\left(18 R^{\circ}\right)-C\left(18^{\circ}\right)$ & $122.3(1)$ & 122.3 \\
\hline 38 & 2) & $1-C\left(4^{*}\right)$ & $119.8(1)$ & $19.2(3)$ & $c\left(1^{\circ}\right)-c\left(2^{\circ}\right)-c\left(3^{\circ}\right)$ & 1 & $121.5(3)$ & $c\left(38^{\circ}\right)-$ & $118.1(1)$ & $117.9(3)$ \\
\hline
\end{tabular}

Molecular Structure of 2: In Figure 4 views of 2 normal and parallel to the naphthalene plane are shown $(A, B)$. In constrast to 1 the spreading of the peri-bonds $C(1)-C\left(1^{\prime}\right)$ and $C(8)-C\left(1^{\prime \prime}\right)$ occurs mainly as an in-plane bending of about $16^{\circ}$. The out-of-plane deviations of these bonds as well as of $C(1)$ and $C(8)$ are negligible. The bond angles $C(1)-C(1)-C(8 A), C(1)-C(8 A)-C(8)$ and $C(8 A)-C(8)-C\left(1^{\prime \prime}\right)$ are widened to $125.2,125.3$, and $124.5^{\circ}$, respectively. The pyrenyl groups show a deviation from planarity for $C\left(1^{\prime}\right)$ and $C\left(1^{\prime}\right)$ [angles of planes $C\left(10 \mathrm{~A}^{\prime}\right) / C\left(1^{\prime}\right) / C\left(2^{\prime}\right)$ and $C\left(10 \mathrm{~A}^{\prime}\right)^{\prime} /$ $\mathrm{C}\left(1^{\prime \prime}\right) / \mathrm{C}\left(2^{\prime \prime}\right)$ with the mean planes through all other pyrenyl carbon atoms: 8.1 and $5.7^{\circ}$. The pyrene planes are twisted against the naphthalene plane for $75^{\circ}$ both; the inclination of the two pyrene planes against each other is $6.8^{\circ}$. There are short interplanar distances only between the ring of carbon atoms $1^{\prime}, 2^{\prime}, 3^{\prime}, 3 \mathrm{~A}^{\prime}, 3 \mathrm{~B}^{\prime}$, and $10 \mathrm{~A}^{\prime}$ and the corresponding ring of the neighbouring pyrene (Table 9). The overlap for the other regions of the pyrene units is very small, and in contrast to 1 it cannot be 
increased considerably by changes of torsional angles. Bond lengths and bond angles of 2 are listed in Tables 7 and 8.

A
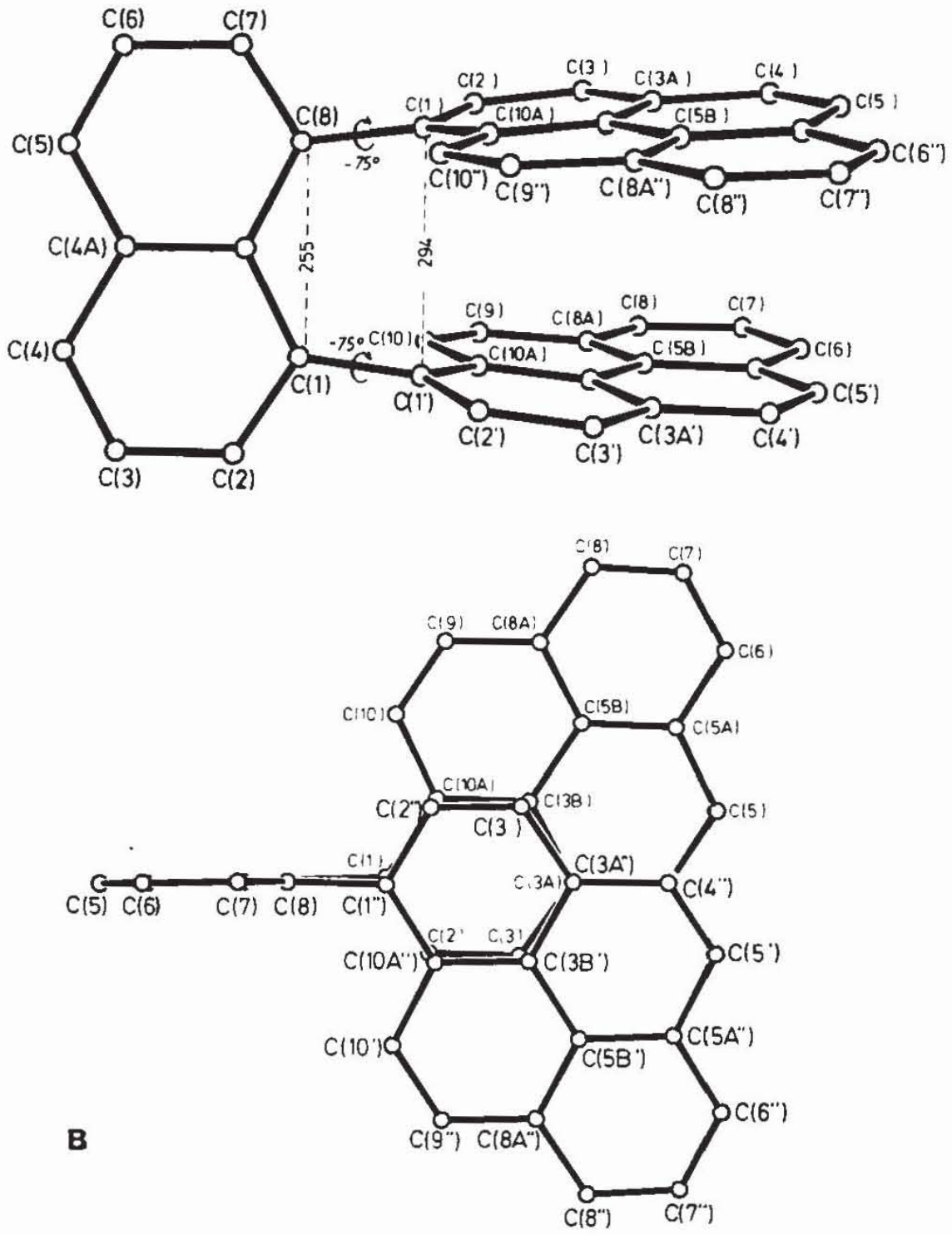

Figure 4. Molecular Structure of $\mathbf{2}$ in a View Normal to the Naphthalene Plane (A) and in a View Parallel to the Naphthalene Plane (B)

Table 9. Some Short Distances in 2 between Carbon Atoms of Different Pyrene Units (in pm)

\begin{tabular}{|c|c|c|c|c|c|}
\hline$C\left(1^{\prime}\right)$ & $\cdots \mathrm{C}\left(1^{\prime \prime}\right)$ & 294 & $C\left(2^{\prime}\right)$ & $\cdots C\left(10 A^{\prime \prime}\right)$ & \\
\hline $\mathrm{C}(1)$ & $\cdots C\left(10 A^{\prime}\right)$ & 313 & $C(1)$ & $\cdots C\left(10^{\prime}\right)$ & \\
\hline$C(10 A$ & $\cdots \mathrm{C}\left(1^{\prime \prime}\right)$ & 314 & $C\left(10^{\prime}\right)$ & $\cdots C\left(1^{\prime \prime}\right)$ & \\
\hline $\mathrm{C}(10 \mathrm{~A}$ & $\cdots C\left(2^{\prime \prime}\right)$ & 319 & $C\left(3 B^{\prime}\right)$ & $\cdots C\left(3^{\prime \prime}\right)$ & \\
\hline$C\left(10^{\prime}\right)$ & $\cdots \mathrm{C}\left(2^{\prime \prime}\right)$ & 320 & $C(3)$ & $\cdots C\left(3 B^{\prime \prime}\right)$ & \\
\hline$C\left(2^{\prime}\right)$ & $\cdots \mathrm{C}\left(10^{\prime}\right)$ & 321 & $C\left(3 A^{\prime}\right)$ & $\cdots C\left(3 B^{\prime}\right)$ & \\
\hline
\end{tabular}

Molecular Structure of 3: In Figure 5A a view is given onto the naphthalene plane which by rotation around the $C(4 A)-C(8 A)$ axis forms an angle with the drawing plane of $40^{\circ}$. In Figure $5 B$ the structure is shown in a view parallel to the naphthalene plane. The in-plane and out-of-plane deformations of the naphthalene peri-bonds Chem. Ber. 117 (1984) 


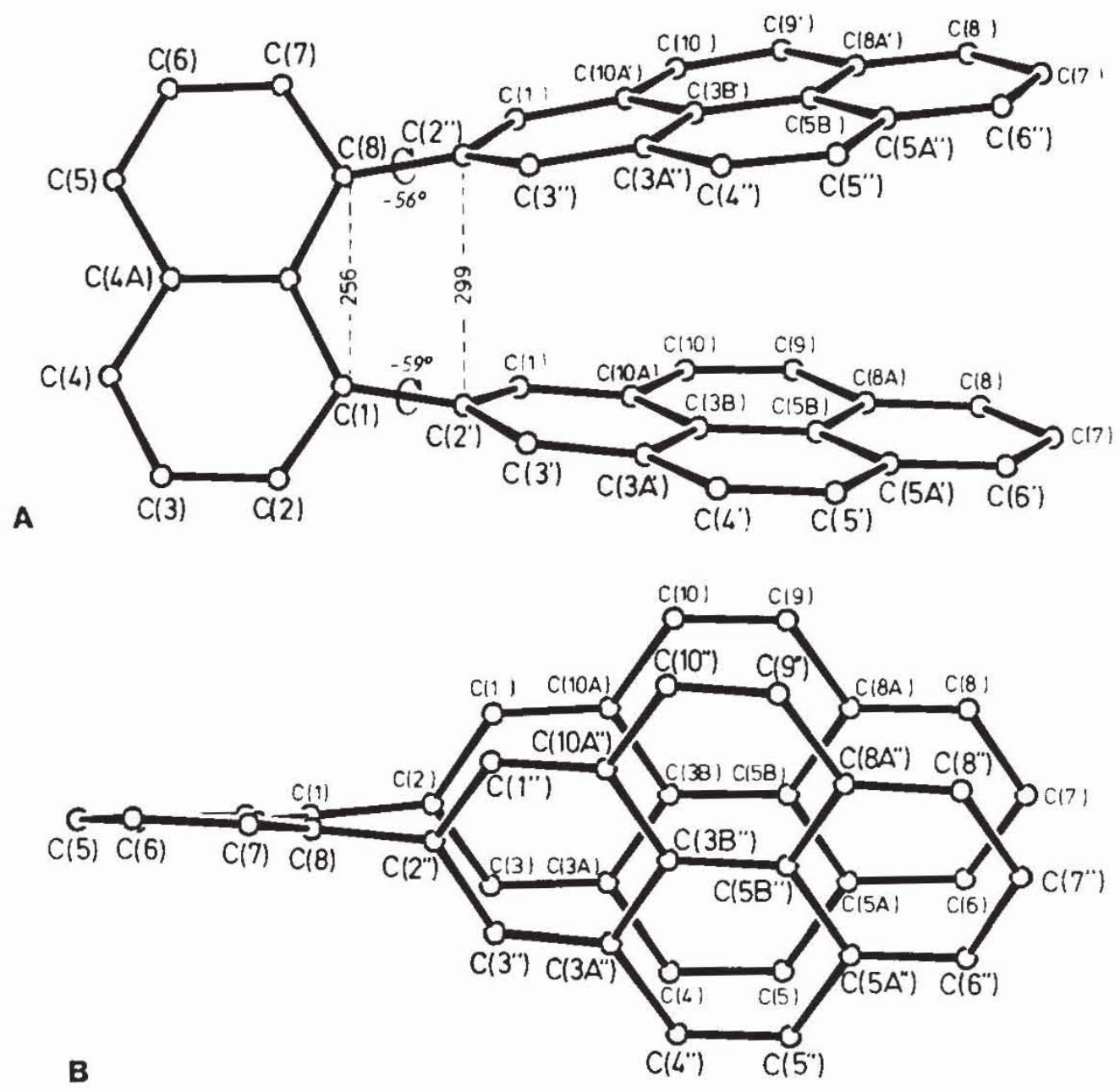

Figure 5. Molecular Structure of 3 in a View onto the Naphthalene Plane (A) and in a View Parallel to the Naphthalene Plane (B)

Table 10. Some Short Distances between Carbon Atoms of Different Pyrene Units in 3 (in pm)

$\begin{array}{lllll}\mathrm{C}\left(1^{\prime}\right) \cdots \mathrm{C}\left(2^{\prime}\right) & 292 & \mathrm{C}\left(1^{\prime}\right) & \cdots \mathrm{C}\left(1^{\prime \prime}\right) & 326 \\ \mathrm{C}\left(2^{\prime}\right) \cdots \mathrm{C}\left(3^{\prime \prime}\right) & 296 & \mathrm{C}\left(3 \mathrm{~B}^{\prime}\right) & \cdots \mathrm{C}\left(3 \mathrm{~A}^{\prime \prime}\right) & 342 \\ \mathrm{C}\left(2^{\prime}\right) \cdots \mathrm{C}\left(2^{\prime}\right) & 299 & \mathrm{C}\left(10^{\prime}\right) \cdots C\left(10 \mathrm{~A}^{\prime \prime}\right) & 346 \\ \mathrm{C}\left(1^{\prime}\right) \cdots \mathrm{C}\left(3^{\prime \prime}\right) & 318 & \mathrm{C}\left(3^{\prime}\right) \cdots C \mathrm{C}\left(4^{\prime \prime}\right) & 347 \\ \mathrm{C}\left(3^{\prime}\right) \cdots \mathrm{C}\left(3^{\prime \prime}\right) & 323 & \mathrm{C}\left(10 \mathrm{~A}^{\prime}\right) \cdots \mathrm{C}\left(3 \mathrm{~A}^{\prime \prime}\right) & 350\end{array}$

$C(1)-C\left(1^{\prime}\right)$ and $C(8)-C\left(1^{\prime \prime}\right)$ are similar as in 1 (16 and $10^{\circ}$, respectively). The angles $\mathrm{C}\left(2^{\prime}\right)-\mathrm{C}(1)-\mathrm{C}(8 \mathrm{~A}), \mathrm{C}(1)-\mathrm{C}(8 \mathrm{~A})-\mathrm{C}(8)$, and $\mathrm{C}(8 \mathrm{~A})-\mathrm{C}(8)-\mathrm{C}\left(2^{\prime \prime}\right)$ are widened to $123.6,126.2$, and $126.3^{\circ}$, respectively. Of the pyrene units $C\left(2^{\prime}\right)$ and $C\left(2^{\prime \prime}\right)$ deviate from planarity [angles of planes $\mathrm{C}\left(1^{\prime}\right) / \mathrm{C}\left(2^{\prime}\right) / \mathrm{C}\left(3^{\prime}\right)$ and $\mathrm{C}\left(1^{\prime \prime}\right) / \mathrm{C}\left(2^{\prime \prime}\right) / \mathrm{C}\left(3^{\prime \prime}\right)$ with the mean plane through all other pyrene carbon atoms: 6.8 and $5.1^{\circ}$, respectively]; for the remaining carbon atoms the maximal deviations from planarity are $6 \mathrm{pm}$ only. As the most interesting feature in the structure of $\mathbf{3}$ it is found that the pyrene planes are not nearly orthogonal to the naphthalene plane but form torsional angles of only 56 and $59^{\circ}$, respectively. This twisting of the pyrene planes results in a substantial reduction of overlap as compared to an ecliptic arrangement of corresponding pyrene carbon atoms. On the other hand, as for $\mathbf{1}$ by an increase of torsional angles only a more extended 
हิ

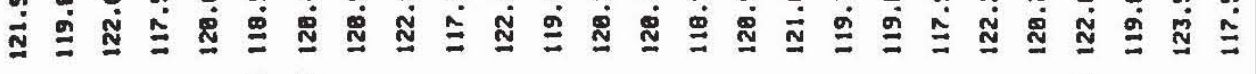

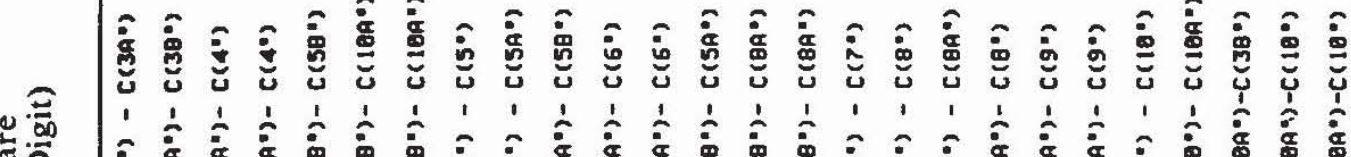

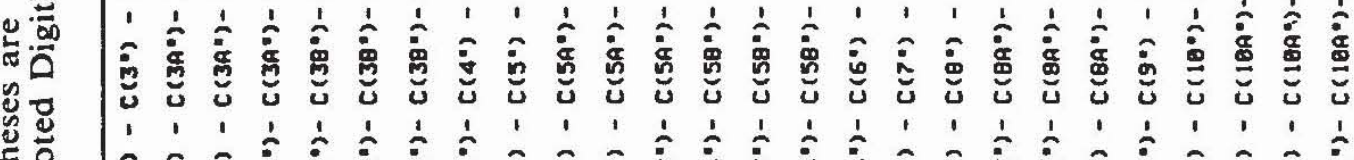

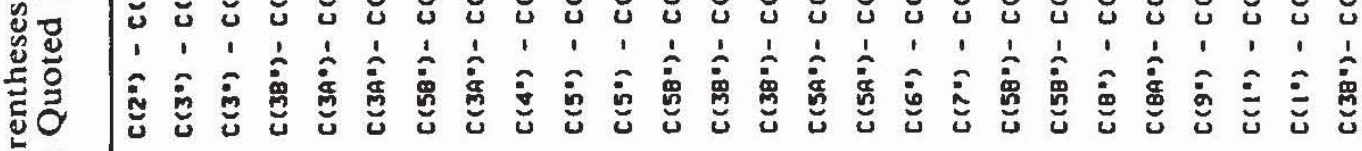
苟

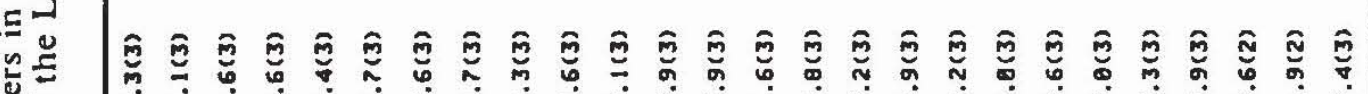
范

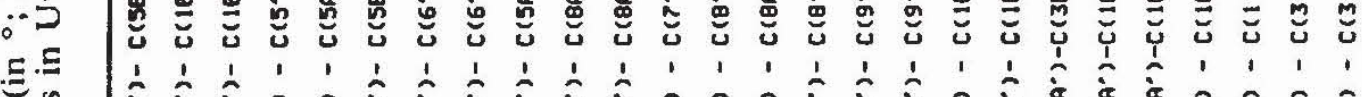

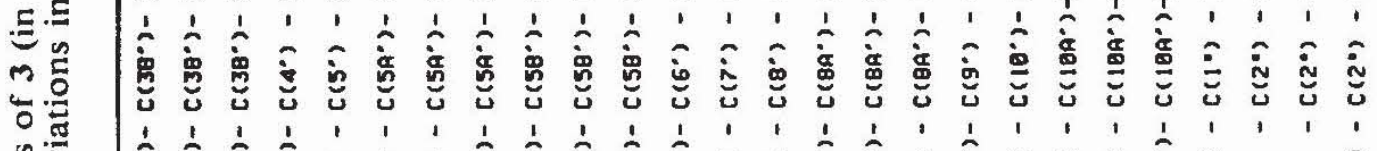

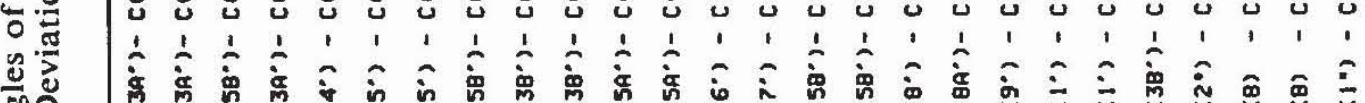
造

뭉ำ

¿

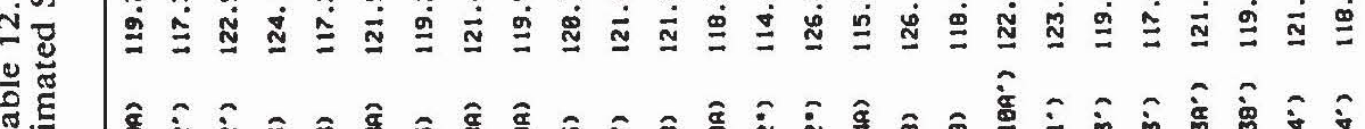

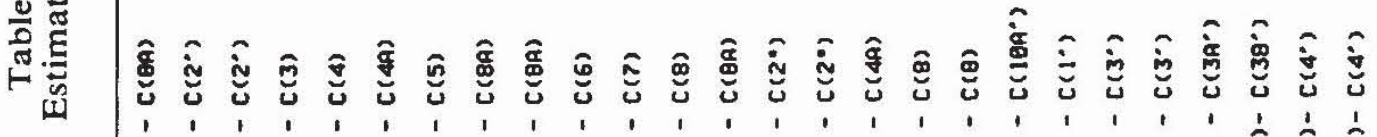
引

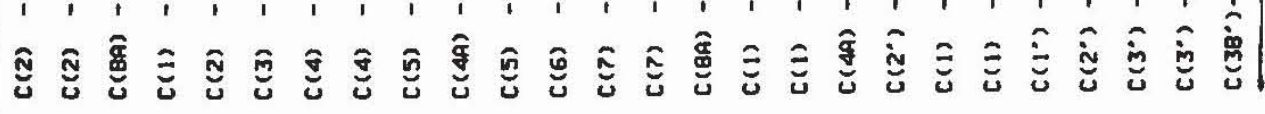

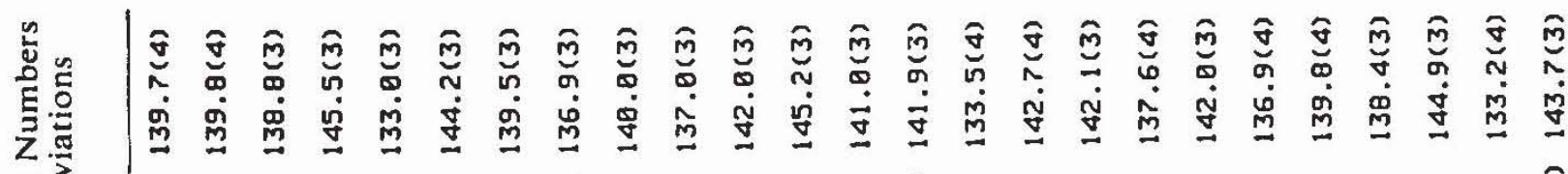

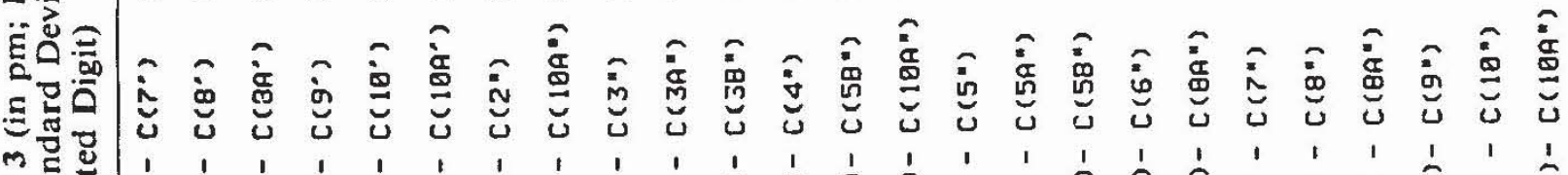

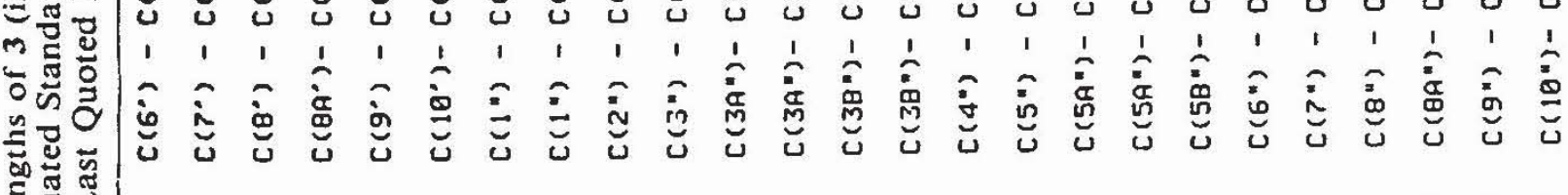
造芯芯

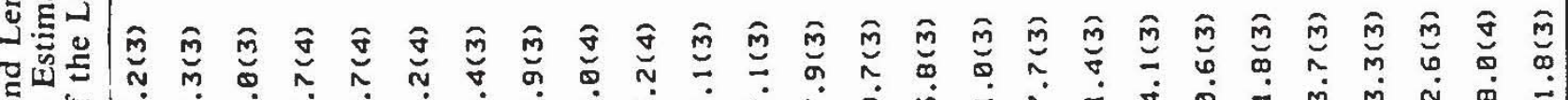

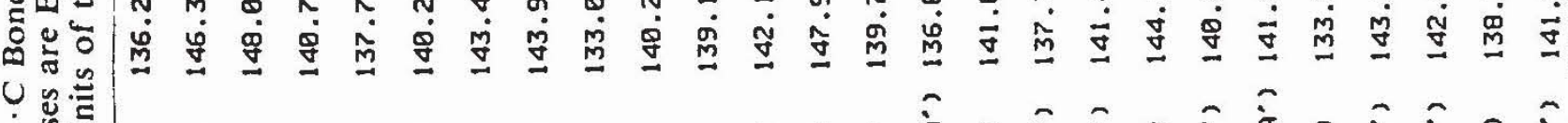

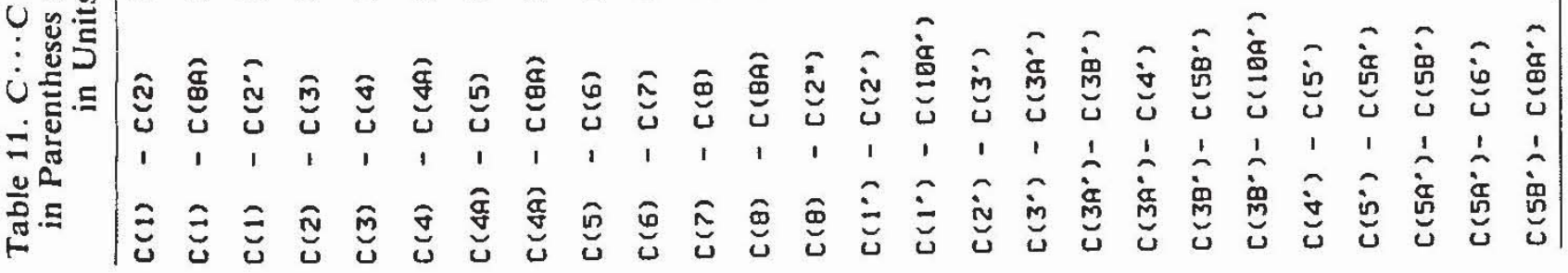


overlap of the pyrene units can easily be reached. Some short distances between carbon atoms of different pyrene units are listed in Table 10. Bond lengths of $\mathbf{3}$ are quoted in Table 11, bond angles in Table 12.

Conclusion: With regard to pyrene-pyrene interactions in 1, 2, and 3 the X-ray structure analyses are in an excellent accordance with the emission spectra discussed above. Thus, the dependence of the formation of an 'excimer-like' state on the mutual overlap of the pyrene units in $\mathbf{1 - 3}$ is strongly supported.

We thank Ms. B. Eberle for very helpful technical assistance. Support of this project by Stiftung Volkswagenwerk, Hannover, and Fonds der Chemischen Industrie, Frankfurt/Main, is gratefully acknowledged.

\section{Experimental Part}

cis- and trans-1,8-Di(1-pyrenyl)naphthalenes $(1,2): 630 \mathrm{mg}(26 \mathrm{mmol})$ of magnesium and $50 \mathrm{mg}$ of iodine in $5 \mathrm{ml}$ of ether were stirred under argon in an ultrasonic bath for $5 \mathrm{~min}$. After evaporation of the ether about one tenth of a solution of $5.1 \mathrm{~g}(18.2 \mathrm{mmol})$ of 1-bromopyrene ${ }^{3)}$ in $50 \mathrm{ml}$ of tetrahydrofuran was added, and the reaction was started by addition of $0.25 \mathrm{~g}$ of dibromoethane and immersion of the reaction flask into an ultrasonic bath of $60^{\circ} \mathrm{C}$. Under these conditions the remaining solution of 1-bromopyrene was dropped in within $20-30 \mathrm{~min}$, and the reaction mixture was stirred for further $10-20 \mathrm{~min}$. The solution of the Grignard reagent then was added dropwise through a glass-wool filter to a solution of $1.14 \mathrm{~g}(3.0 \mathrm{mmol})$ of 1,8-diiodonaphthalene ${ }^{4)}$ and $40 \mathrm{mg}$ of bis(acetylacetonato)nickel(II) in $20 \mathrm{ml}$ of tetrahydrofuran. After warming up to room temperature within $2-3 \mathrm{~h}$, further stirring at room temperature for $2 \mathrm{~h}$ and hydrolysis with conc. ammonium chloride solution, tetrahydrofuran was evaporated $ı$ vacuo. To the residue $50 \mathrm{ml}$ of ethanol was added, and the solution was poured into $200 \mathrm{ml}$ of water. The precipitate was filtered off, dried in vacuo and extracted with $300 \mathrm{ml}$ of dichloromethane. The insoluble material was filtered off ( $150 \mathrm{mg}$, predominantly $1,1^{\prime}$-bipyrene). The soluble products were chromatographed from toluene at basic aluminium oxide (activity grade I - II); in the order of decreasing $R_{\mathrm{F}}$-values the following compounds were isolated:

I-(l-Pyrenyl)naphthalene (4): $90 \mathrm{mg}, \mathrm{m}$. p. $164-166^{\circ} \mathrm{C}$ (from acetone), identified with an authentic sample (see below).

1,1'-Bipyrene: $320 \mathrm{mg}, \mathrm{m}$. p. $323-326^{\circ} \mathrm{C}$ (from xylene; lit. ${ }^{5)} \mathrm{m}$. p. $327-328^{\circ} \mathrm{C}$ ).

1,8-Di(1-pyrenyl)naphthalenes (1/2): $420 \mathrm{mg}$ (26.5\%) (for separation of 1 and 2 see below).

8, ' 'Di(1-pyrenyl)-1,1'-binaphthalene (5): $30 \mathrm{mg}$; m. p. $375^{\circ} \mathrm{C}$ (dec.). - ${ }^{1} \mathrm{H}$ NMR $(360 \mathrm{MHz}$, $\left[\mathrm{D}_{2}\right]$-1,1,2,2-tetrachloroethane): $\delta=5.26$ ('dd', $2 \mathrm{H}$ ), 6.37 ('dd', $2 \mathrm{H}$ ), 6.48 ('dd', $2 \mathrm{H}$ ), 6.76 (d, $J=9.1 \mathrm{~Hz}, 2 \mathrm{H}), 7.31$ (dd, $J=7.0$ and $1.3 \mathrm{~Hz}, 2 \mathrm{H}), 7.35(\mathrm{~d}, J=7.8 \mathrm{~Hz}, 2 \mathrm{H}), 7.43(\mathrm{dd}, J=8.1$ and $1.3 \mathrm{~Hz}, 2 \mathrm{H}), 7.47-7.49(\mathrm{~m}, 4 \mathrm{H}), 7.53(\mathrm{~d}, J=9.3 \mathrm{~Hz}, 2 \mathrm{H}), 7.84(\mathrm{~d}, J=9.0 \mathrm{~Hz}, 2 \mathrm{H})$, $7.92-8.01(\mathrm{~m}, 4 \mathrm{H}), 8.03(\mathrm{~d}, J=8.0 \mathrm{~Hz}, 2 \mathrm{H}), 8.14(\mathrm{~d}, J=6.8 \mathrm{~Hz}, 2 \mathrm{H}) .-\mathrm{MS}: m / z=654$ $\left(100 \%, \mathrm{M}^{+}\right), 327(8 \%)$.

$$
\mathrm{C}_{52} \mathrm{H}_{30} \text { (654.8) Calcd. C } 95.38 \text { H } 4.62 \text { Found C } 95.51 \mathrm{H} 4.63
$$

Separation of cis-trans-Isomers 1 and 2: The 1/2-fraction from the above-mentioned chromatography was adsorbed on silica (particle size $<0.063 \mathrm{~mm}$ ) from dichloromethane. By pressure-chromatography ( $3-5 \mathrm{bar}$ ) from tetrachloromethane $260 \mathrm{mg}(16 \%)$ of 1 with $R_{\mathrm{F}}=0.2$ and $150 \mathrm{mg}(9.5 \%)$ of 2 with $R_{\mathrm{F}}=0.1$ were obtained. Crystallization of 1 and 2 was achieved by dissolving the respective compound in dichloromethane at room temperature, adding toluene, and slowly evaporating dichloromethane at room temperature. 
1: pale-yellow crystals, m. p. $267-269^{\circ} \mathrm{C}$ (with partial conversion to 2 ); for spectroscopic data see above.

2: colourless crystals, m. p. $262-266^{\circ} \mathrm{C}$ (with partial conversion to 1 ); for spectroscopic data see above.

$$
\begin{array}{rrrr}
\mathrm{C}_{42} \mathrm{H}_{24}(528.6) & \text { Calcd. C } 95.42 \mathrm{H} 4.58 & \text { 1: Found } \mathrm{C} 95.69 & \mathrm{H} 4.43 \\
& & \text { 2: Found C } 95.61 & \mathrm{H} 4.62
\end{array}
$$

1-(1-Pyrenyl)naphthalene (4): To a solution of $1.0 \mathrm{~g}$ (3.05 mmol) of 1-iodopyrene ${ }^{7)}$ and $40 \mathrm{mg}$ of bis(acetylacetonato)nickel(II) in $30 \mathrm{ml}$ of tetrahydrofuran, cooled to $-15^{\circ} \mathrm{C}$, the Grignard solution prepared from $2.5 \mathrm{~g}(12.1 \mathrm{mmol})$ of 1-bromonaphthalene in $40 \mathrm{ml}$ of tetrahydrofuran was dropped in within $1 \mathrm{~h}$. The reaction mixture was stirred at $-10^{\circ} \mathrm{C}$ for $1 \mathrm{~h}$ and then hydrolysed with diluted hydrochloric acid. Tetrahydrofuran was evaporated, the residue was extracted with dichloromethane; the extract was washed with water, dried over sodium sulfate, and chromatographed on silica from tetrachloromethane. Following the fraction of naphthalene $500 \mathrm{mg}$ of 1,1'-binaphthalene (m. p. $152-153^{\circ} \mathrm{C}$, lit. ${ }^{11)} 155^{\circ} \mathrm{C}$ ) and $620 \mathrm{mg}(62 \%)$ of 4 were obtained: colourless crystals, m. p. $164-166^{\circ} \mathrm{C}$ (from acetone). - MS: $m / z=328\left(100 \%, \mathrm{M}^{+}\right), 326(60), 324(10)$, $164\left(14, \mathrm{M}^{2+}\right)$ a. 0 .

$$
\mathrm{C}_{26} \mathrm{H}_{16} \text { (328.4) Calcd. C } 95.09 \text { H } 4.91 \text { Found C } 95.24 \text { H } 4.78
$$

2-Bromo-4,5,9,10-tetrahydropyrene (6): The synthesis of 6 from [2.2]metacyclophane (yield $15 \%)^{8)}$ has been modified as follows: To a solution of $10.4 \mathrm{~g}(50 \mathrm{mmol})$ of [2.2]metacyclophane and $200 \mathrm{mg}$ of iodine in $150 \mathrm{ml}$ of tetrachloromethane under ice-cooling a solution of $16 \mathrm{~g} \mathrm{(100}$ mmol) of bromine in $100 \mathrm{ml}$ of tetrachloromethane was added within $1 \mathrm{~h}$. The solvent was evaporated, the residue dissolved in dichloromethane and filtered through basic aluminum oxide (activity grade I): $12.8 \mathrm{~g}(90 \%)$, m. p. $94-96^{\circ} \mathrm{C}$ (lit. ${ }^{8)} 97.5-100^{\circ} \mathrm{C}$ ); the product is sufficiently pure for the following reaction.

1,8-Bis(4,5,9,10-tetrahydro-2-pyrenyl)naphthalene (7): A Grignard solution prepared from $5.2 \mathrm{~g}(18.2 \mathrm{mmol})$ of 6 as described for the $1 / 2$-synthesis was added dropwise within $1.5 \mathrm{~h}$ to a solution of $1.14 \mathrm{~g} \mathrm{(3.0} \mathrm{mmol)} \mathrm{of} \mathrm{1,8-diiodonaphthalene} \mathrm{and} 50 \mathrm{mg}$ of bis(acetylacetonato)nickel(II) in $40 \mathrm{ml}$ of tetrahydrofuran cooled to -15 to $-10^{\circ} \mathrm{C}$. The reaction mixture was stirred for $1 \mathrm{~h}$ at $-10^{\circ} \mathrm{C}$, warmed up to room temperature within $1 \mathrm{~h}$ and hydrolysed with diluted hydrochloric acid. The solution was evaporated to about $20 \mathrm{ml}$, mixed with $100 \mathrm{ml}$ of water and extracted three times with $50 \mathrm{ml}$ of dichloromethane. The extract was washed with water, dried over sodium sulfate, and evaporated. The residue was filtered through basic aluminum oxide from toluene and then chromatographed on silica $(<0.063 \mathrm{~mm})$ from tetrachloromethane $(3-5$ bar). Following a tetrahydropyrene fraction $260 \mathrm{mg}$ of compound A (see below) and $350 \mathrm{mg}$ $(22 \%)$ of 7 were obtained. 7 forms colourless crystals, m. p. $281-283^{\circ} \mathrm{C}$ (from toluene). ${ }^{1} \mathrm{H}$ NMR ( $\left.360 \mathrm{MHz}, \mathrm{CD}_{2} \mathrm{Cl}_{2}\right): \delta=2.4-2.75(\mathrm{~m}, 16 \mathrm{H}), 6.727(\mathrm{~s}, 4 \mathrm{H}), 6.840$ ('d', $\left.4 \mathrm{H}\right), 6.957$ (m, $2 \mathrm{H}$ ), $7.48-7.58(\mathrm{~m}, 4 \mathrm{H}), 7.935$ ('dd', $J \approx 8.0$ and $1.3 \mathrm{~Hz}, 2 \mathrm{H}$ ).

$$
\mathrm{C}_{42} \mathrm{H}_{32} \text { (536.7) Calcd. C } 93.99 \text { H } 6.01 \text { Found C } 94.17 \text { H } 6.24
$$

Compound $\mathrm{A}$, colourless crystals of $\mathrm{m}$. p. $317-321^{\circ} \mathrm{C}$ (from toluene), was identified as $4,4^{\prime}, 5,5^{\prime}, 9,9^{\prime}, 10,10^{\prime}$-octahydro-2,2'-bipyrene: ${ }^{1} \mathrm{H}$ NMR $\left(80 \mathrm{MHz},\left[\mathrm{D}_{2}\right]-1,1,2,2\right.$-tetrachloroethane): $\delta=2.94(\mathrm{~s}, 16 \mathrm{H}), 7.10(\mathrm{~s}, 6 \mathrm{H}), 7.35(\mathrm{~s}, 4 \mathrm{H}) .-\mathrm{MS}: \mathrm{m} / z=410\left(100 \%, \mathrm{M}^{+}\right), 408(4)$, $205\left(10, \mathrm{M}^{2+}\right)$.

$$
\mathrm{C}_{32} \mathrm{H}_{26} \text { (410.6) Calcd. C } 93.62 \text { H } 6.38 \text { Found C } 93.87 \text { H } 6.34
$$

1,8-Di(2-pyrenyl)naphthalene (3): $100 \mathrm{mg}(0.186 \mathrm{mmol})$ of 7 and $200 \mathrm{mg}(0.881 \mathrm{mmol})$ of 2,3-dichloro-5,6-dicyano-1,4-benzoquinone in $20 \mathrm{ml}$ of toluene were heated under reflux for $1.5 \mathrm{~h}$. The cooled reaction mixture was diluted with dichloromethane and filtered through a short column of

Chem. Ber. 117 (1984) 
aluminum oxide. Evaporation of the solvents yielded $82 \mathrm{mg}(83 \%)$ of 3, pale-yellow crystals, m.p. $331-333^{\circ} \mathrm{C}$; for spectroscopic data see above.

$$
\mathrm{C}_{42} \mathrm{H}_{24} \text { (528.6) Calcd. C } 95.42 \mathrm{H} 4.58 \text { Found C } 95.55 \text { H } 4.34
$$

1-(2-Pyrenyl)naphthalene (8): Preparation from $1.0 \mathrm{~g} \mathrm{(3.05} \mathrm{mmol)} \mathrm{of} \mathrm{2-iodopyrene}{ }^{7)}$ and the Grignard solution prepared from $2.5 \mathrm{~g}(12.1 \mathrm{mmol})$ of 1-bromonaphthalene following exactly the procedure mentioned for 4 : yield $540 \mathrm{mg}(54 \%)$, colourless crystals (from isopropyl alcohol), m. p. $123-124.5^{\circ} \mathrm{C}$ (lit. $\left.{ }^{9)} 112-113^{\circ} \mathrm{C}\right)$. - MS: $m / z=328(100 \%), 326(50), 324(8), 164(14$, $\mathrm{M}^{2+}$ ), a. o.

$$
\mathrm{C}_{26} \mathrm{H}_{16} \text { (328.4) Calcd. C } 95.09 \text { H } 4.91 \text { Found C } 95.21 \text { H } 4.86
$$

1) H. A. Staab, N. Riegler, F. Diederich, C. Krieger, and D. Schweitzer, Chem. Ber. 117, 246 (1984), preceding.

2) R. L. Clough, P. Mison, and J. D. Roberts, J. Org. Chem. 41, 2252 (1976).

3) R. H. Mitchell, Y.-H. Lai, and R. V. William, J. Org. Chem. 44, 4733 (1979).

4) H. O. House, D. G. Koepsell, and W. J. Campbell, J. Org. Chem. 37, 1003 (1972).

5) E. Clar and O. Kühn, Liebigs Ann. Chem. 601, 181 (1956).

6) R. L. Clough and J. D. Roberts, J. Am. Chem. Soc. 98, 1018 (1976); see also E. Ibuki, S. Ozasa, Y. Fujioka, and H. Mizutanl, Chem. Pharm. Bull. 29, 2103 (1981); Bull. Chem. Soc. Jpn. 55, 845 (1982).

7) A. Jensen and A. Berg, Acta Chem. Scand. 19, 520 (1965).

8) N. L. Allinger, B. J. Gorden, Shih-En Hu, and R. A. Ford, J. Org. Chem. 32, 2272 (1967).

9) Ch. Jutz, R. Kirchlechner, and H.-J. Seidel, Chem. Ber. 102, 2301 (1969).

${ }^{10)}$ Further information concerning the $X$-ray structure analyses of 1,2 , and 3 may be requested from Fachinformationszentrum Energie Physik Mathematik, D-7514 Eggenstein-Leopoldshafen, with indication of the registry number CSD 50429, the authors' names, and the reference to this publication.

11) F. Ullmann and J. Bielecki, Ber. Dtsch. Chem. Ges. 34, 2184 (1901).

$[120 / 83]$ 